\title{
Fourth order gravity: equations, history, and applications to cosmology*
}

\author{
Hans-Jürgen Schmidt \\ 42nd Karpacz Winter School, February 6 - 11, 2006 \\ Universität Potsdam, Institut für Mathematik, Am Neuen Palais 10 \\ D-14469 Potsdam, Germany, E-mail: hjschmi@rz.uni-potsdam.de \\ http://www.physik.fu-berlin.de/ ${ }^{\circ}$ hsschmi
}

\begin{abstract}
The field equations following from a Lagrangian $L(R)$ will be deduced and solved for special cases. If $L$ is a non-linear function of the curvature scalar, then these equations are of fourth order in the metric. In the introduction we present the history of these equations beginning with the paper of $\mathrm{H}$. Weyl from 1918, who first discussed them as alternative to Einstein's theory. In the third part, we give details about the cosmic no hair theorem, i.e., the details how within fourth order gravity with $L=R+R^{2}$ the inflationary phase of cosmic evolution turns out to be a transient attractor. Finally, the Bicknell theorem, i.e. the conformal relation from fourth order gravity to scalar-tensor theory, will be shortly presented.
\end{abstract}

*This paper is a shortened version of my (still unpublished) "Lectures on Mathematical Cosmology", see its preprint version gr-qc/0407095. Parts of my paper with R. Schimming "On the history of fourth order metric theories of gravitation" NTM-Schriftenr. Gesch. Naturw., Tech., Med. (Leipzig) 27 (1990) 41, gr-qc/0412038, are incorporated. 


\section{To the history of fourth order gravity}

From the advent of the general relativity theory (GRT) in 1915 by Albert Einstein (1879-1955) until today numerous geometrized theories of gravitation have been proposed. Here, we shall review the history of a class of theories which is conceptually rather close to GRT:

- The gravitational field is described by a space-time metric only.

- The field equation follows from a Hamiltonian principle. The Lagrangian

$L$ is a quadratic scalar in die Riemannian curvature of the metric.

- The constants appearing in this ansatz are adjusted such that the theory is compatible with experimentally established facts. Hence, the Lagrange function reads

$$
L=a R^{2}+b R_{i j} R^{i j}+k R+\Lambda
$$

with constants $a, b, k, \Lambda$ where $a$ and $b$ do not vanish simultaneously. The variational derivative of $R_{i j k l} R^{i j k l}$ with respect to the metric can be linearly expressed by the variational derivatives of $R_{i j} R^{i j}$ and of $R^{2}$ [1]. Thus we may omit $R_{i j k l} R^{i j k l}$ in (1.1) without loss of generality. The theory is scaleinvariant if and only if $\Lambda \cdot k=0$. It is even conformally invariant if and only if $\Lambda=k=0$ and $3 a+b=0$. The field equation following from $L$ eq. (1.1) is of fourth order, i.e. it contains derivatives up to the fourth order of the components of the metric with respect to the space-time coordinates.

The fourth order metric theories of gravitation are a very natural modification of the GRT. Historically, they have been introduced as a specialization of Hermann Weyl's (1885-1955) nonintegrable relativity theory from 1918 [2]. Later on, just the fourth order theories became interesting and more and more physical motivations supported them: The fourth order terms can prevent the big bang singularity of GRT; the gravitational potential of a point mass is bounded in the linearized case; the inflationary cosmological model is a natural outcome of this theory. But all the arguments from classical physics were not so convincing as those from quantum physics: the quantization of matter fields with unquantized gravity background leads to a gravitational Lagrangian of the above form [3]. Moreover, fourth order theories turned out to be renormalizable at the one-loop quantum level [4], but at the price of losing the unitarity of the S-matrix. These circumstances caused a boom 
of fourth order gravity (classical as well as quantum) in the seventies. We will stop our record of the history before this boom. We restrict ourselves to the purely metrical theories (i.e., the affinity is always presumed to be Levi-Civita) and want only to mention here that fourth order field equations following from a variational principle can be formulated in scalar-tensor theories, theories with independent affinity, and other theories alternative to GRT as well.

\subsection{Papers inspired by Weyl's theory}

In 1918, soon after Albert Einstein's proclamation of the GRT, Hermann Weyl proposed a new kind of geometry and a unified theory of gravitation and electromagnetism based on it. He dwelled on the matter in a series of papers [2, 5-10] until it became superseded by the modern gauge field interpretation of electromagnetism [11-13]. Note that the gauge concept together with the words "Eichung" (gauge) and "Eichinvarianz" (gauge invariance) came into use in theoretical physics through Weyl's ansatz. For a broader discussion and evaluation we refer to [14]. A. Einstein [15] pointed out that the nonintegrability of the lengths of vectors under Weyl-like parallel propagation contradicts to physical experience. His argument has been refuted not earlier than in 1973: P. Dirac discusses the possibility of a varying gravitational constant. He writes:

"Such a variation would force one to modify Einstein's theory of gravitation. It is proposed that the modification should consist in the revival of Weyl's geometry, in which lengths are nonintegrable when carried around closed loops, the lack of integrability being connected with the electromagnetic field". [16, p. 403]

H. Weyl's aesthetically very appealing modification of GRT unfortunately does not directly describe the real dynamics of fields and particles; however it deeply influenced the "dynamics of theories". By this we mean that various fundamental ideas have been formed or promoted by Weyl's papers:

- the search for alternatives to the GRT based on geometrization;

- the unification of the interactions or forces of nature, beginning with gravity and electromagnetism; 
- field theories based on the geometry of an affine connection;

- conformal geometry and conformally invariant field theories;

- the gauge field idea, and

- fourth order gravitational field equations.

Here we are interested just in the last item. Weyl required the Lagrangian to be a polynomial function of the curvature and to be conformally invariant. He states: "Dies hat zur Folge, dass unsere Theorie wohl auf die Maxwellschen elektromagnetischen nicht aber auf die Einsteinschen Gravitationsgleichungen führt; an ihre Stelle treten Differentialgleichungen 4. Ordnung." [2, p. 477]

The ambiguity in the concrete choice of $L$ appeared as a difficulty which is opposed to the spirit of unification: any linear combination of $R^{2}$ and $R_{i j} R^{i j}$ would do. The variation of $R_{i j} R^{i j}$ or of $R_{i j k l} R^{i j k l}$ with respect to the vector field yields Maxwell-like equations, while for the choice of $R^{2}$ an electromagnetic Lagrangian $F_{i j} F^{i j}$ together with a coupling constant $\alpha$ has to be added by hand: $L=R^{2}+\alpha F_{i j} F^{i j}[6,2]$. Weyl himself favoured different Lagrangians in different papers. Moreover, he took trouble to produce results compatible with Einstein's GRT. For this aim he destroyed the conformal invariance by a special gauge. Ernst Reichenbächer criticizes: "Um so auffallender ist es, dass Weyl in dem von ihm durchgerechneten Beispiel für die Wirkungsfunktion durch Festlegung der Eichung vor der Variation den Grundsatz der Eichinvarianz durchbricht." [17, p. 157]. A more detailed analysis of the theory was necessary then. R. Weitzenböck [18] produced and studied all scalar invariants of the curvature in Weyl's geometry. W. Pauli $[19,20]$ and a little later F. Jüttner [21] calculated the spherically symmetric static gravitational field for variants of Weyl's theory.

Pauli [20, S. 748] comes to an important conclusion: "Hiernach ist klar, dass aus Beobachtungen der Merkurperihelbewegung und der Strahlenablenkung, die mit Einsteins Feldgleichungen im Einklang sind, niemals ein Argument gegen Weyls Theorie entnommen werden kann, wenigstens solange die letztere eine der drei Invarianten $R_{i j} R^{i j}, R^{2}, R_{i j k l} R^{i j k l}$ als Weltfunktion zugrunde legt."

In other words, fourth order gravitational field equations following from (1.1) are not falsifiable by experimental physics! Pauli [20, 22] and other 
authors did not even consider the vector field (i.e. assumed it to be equal to zero) in Weyl's theory, thus making it unaffected by the criticism of nonintegrability [15]. R. Bach [23] realized the possibility to keep the conformal invariance in a purely metrical theory: a Lagrangian $L=C_{i j k l} C^{i j k l}$ or equivalently, $L=R^{2}-3 R_{i j} R^{i j}$ yields a conformally invariant field equation for the metric, later on called "Bach's equation". In a similar spirit and in the same year 1921, Albert Einstein [24] proposed a conformally invariant theory. His expressions suffer from being non-rational in the metric. This theory is sometimes cited but has never been studied in details.

Reichenbächer [25, 17] proposed a variant of Weyl's theory based on a non-rational Lagrangian resembling nonlinear Born-Infeld electrodynamics.

In [26], also $L=R^{2}$ is used to get a field-theoretical model for the electron, but the fourth order terms are lost by an error in the calculations.

C. Lanczos [27] tried a programme of "Electromagnetismus als natürliche Eigenschaft der Riemannschen Geometrie". He also assumed the vector field in Weyl's theory to be zero, but reintroduced it then in an alternative way as a set of Lagrangian multipliers. Unfortunately, Lanczos was, working with hyperbolic differential equations, misled by a formal analogy with elliptic differential equations. He varied the speculations with Lagrangian multipliers in a series of papers [28-34]. To take it positive, many useful mathematical formulas for fourth order theories resulted from Lanczos' work. Particularly, the paper [1] became a "citation classic".

In the twenties, the programme of classical field theory with its two cornerstones geometrization and unification lost some of its attractiveness in virtue of the quickly progressing quantum theory, cf. [35]. Moreover, there were the refutation of Weyl's theory and objections to fourth order equations. Lanczos expressed them as follows:

"Der Grund, weshalb diese Untersuchungen nicht weiter gediehen sind und zu keinem Fortschritt führten, lag an zwei Momenten. Einerseits war es entmutigend, dass man zumindest drei anscheinend gleichwertige Invarianten zur Verfügung hatte: $R^{2}, R_{\alpha \beta} R^{\alpha \beta}, R_{\alpha \beta \gamma \delta} R^{\alpha \beta \gamma \delta}$, ohne ein plausibles Auswahlprinzip zwischen ihnen zu besitzen. Andererseits erscheinen diese Gleichungen, solange man ihre innere Struktur nicht verstehe, als Differentialgleichungen vierter Ordnung für die die $g_{i k}$ von einer Kompliziertheit sind, 
die für jede weitere Schlussfolgerung ungeeignet ist." [27, p.75]

Similarly, Bergmann argues in his text-book [36] that, first, fourth order equations admit too many solutions and, second, their Lagrangian is rather ambiguous. This situation explains why only few papers on fourth order gravitation appeared in the period from the thirties to the sixties and why these did not follow the actual trends at their time. H. A. Buchdahl dealt with the subject in the period 1948-1980. In his papers he covered the following problems:

- Invariant-theoretical considerations continuing those of Weitzenböck and Lanczos [37];

- General expressions for the variational derivatives of Lagrangians built from the curvature and, possibly, its derivatives are obtained [38-42].

- Einstein spaces are solutions of a rather general class of fourth order equations $[43,44]$;

- Static gravitational fields in fourth order theories [45];

- Cosmological solutions in theories where the Lagrangian is a function of the scalar curvature [46, 47];

- Conformal gravity [48];

- Reinterpretation of some fourth order equations in five dimensions [49].

A. S. Eddington in 1921, see [50], and E. Schrödinger in 1948, see [51], also discussed gravitational field equations of fourth order to get field theoretical particle models, i.e., they tried to realize Einstein's particle programme.

\subsection{A new view}

Fourth order metric theories of gravitation have been discussed from 1918 up to now. One original motivation was the scale invariance of the action, a property which does not hold in GRT. Another motivation was the search for a unification of gravity with electromagnetism, which is only partially achieved with the Einstein-Maxwell system. There was no experimental fact contradicting GRT which could give motives for replacing it by a more complicated theory. But a lot of problems appeared:

1. The Lagrangian became ambiguous in sharp contrast to the required unification. 
2. The higher order of the field equation brought mathematical problems (2.1.) in the search for solutions and physical problems (2.2.) for the interpretation of the additional degrees of freedom.

3. The well-founded Newtonian theory of gravitation did not result as the weak-field limit of scale invariant fourth order gravity.

The third problem was the last of these to be realized but the first to be solved, both in 1947: One has to break the scale invariance of the theory by adding the Einstein-Hilbert action to the purely quadratic Lagrangian. Then, up to an exponentially small term, the correct Newtonian limit appears $[52]$.

The original scale invariant theory then, again emerges as the high-energy limit of that sum. The items 1., 2. and the absence of experimental facts contradicting GRT seemed to restrain die research on these theories already in the twenties. Only in 1966 a renewed interest in these theories arose in connection with a semiclassical description of quantum gravity [53-55]. The coefficient of the quadratic term became calculable by a renormalization procedure, thus solving problem 1, at least concerning the vacuum equation. Further, the fact that fourth order gravity is one-loop renormalizable in contrast to GRT; a fact which was realized in 1977, [4] initiated a boom of research. It is interesting to observe that it is just the scale invariance of the curvature squared terms - the original motivation - which is the reason for the renormalizability. Also the latest fundamental theory - the superstring theory - gives in the field theoretical limit (besides other terms) just a curvature-squared contribution to the action $[56,57]$. The use of modern mathematics and computers has led to a lot of results to clarify the structure of the space of solutions thus solving problem 2.1. in the eighties. The more profound problem 2.2 has now three kinds of answers:

a) In spite of the higher order of the differential equation, a prescribed matter distribution plus the $O(1 / r)$-behaviour of the gravitational potential suffice - such as it takes place in Newtonian theory - to determine the gravitational potential for isolated bodies in a unique way for the weak-field slow-motion limit, $[52,53]$

b) the observation that the additional degrees of freedom are just the phases of damped oscillations which become undetectably small during the 
cosmic evolution, and, by the way, can solve the missing mass problem and prevent the singularity problem of GRT [58], and

c) it is supposed that there exist massive gravitons besides the usual massless gravitons known from GRT, but they are very weakly coupled [59].

The last point to be mentioned is the experimental testability: In the recent three years many efforts have been made to increase the accuracy in determining the constants $G, \alpha$ and $l$ if the gravitational potential is assumed (also in other theories than fourth order gravity) to be $G m r^{-1}\left(1+\alpha e^{-r / l}\right)$. The term proportional to $\alpha$, the "fifth force", can be interpreted as the fourth order correction to GRT. Up to now, it has not been possible to exclude $\alpha=0$ by experiments [60-62].

In 1990, we concluded our paper on the history of fourth order gravity by saying: Fourth order gravity theories will remain an essential link between GRT and quantum gravity for a long time. Now, 16 years later, one can prove the correctness of this prediction by looking at references [63-99], [102], [105-

109] which are all deal with a variant of fourth order gravity or its equivalents and its cosmological consequences and which is still far from being complete.

\section{Scalar fields and $f(R)$ cosmology}

Following the paper "Comparing selfinteracting scalar fields and $R+R^{3}$ cosmological models", gr-qc/0106035, see [82], we generalize the well-known analogies between $m^{2} \phi^{2}$ and $R+R^{2}$ theories to include the self-interaction $\lambda \phi^{4}$-term for the scalar field. It turns out to be the $R+R^{3}$ Lagrangian which gives an appropriate model for it. Considering a spatially flat Friedmann cosmological model, common and different properties of these models are discussed, e.g., by linearizing around a ground state the masses of the corresponding spin 0-parts coincide. Then we prove a general conformal equivalence theorem between a Lagrangian $L=L(R), L^{\prime} L^{\prime \prime} \neq 0$, and a minimally coupled scalar field in a general potential. This theorem was independently deduced by several persons, and it is now known as Bicknell theorem [68]. 


\subsection{Introduction to scalar fields}

For the gravitational Lagrangian

$$
L=\left(R / 2+\beta R^{2}\right) / 8 \pi G,
$$

where $\beta$ is some free but constant parameter, the value

$$
R=R_{\text {crit }}=-1 / 4 \beta
$$

is the critical value of the curvature scalar. It is defined by

$$
\partial L / \partial R=0
$$

In regions where

$$
R / R_{\text {crit }}<1
$$

holds, we can define

$$
\psi=\ln \left(1-R / R_{\text {crit }}\right)
$$

and

$$
\tilde{g}_{i j}=\left(1-R / R_{\text {crit }}\right) g_{i j}
$$

In units where $8 \pi G=1$ we now obtain from the Lagrangian eq. (2.1) via the conformal transformation eq. (2.6) the transformed Lagrangian

$$
\tilde{L}=\tilde{R} / 2-3 \tilde{g}^{i j} \psi_{; i} \psi_{; j} / 4-\left(1-e^{-\psi}\right)^{2} / 16 \beta
$$

being equivalent to $L$, cf. Whitt [85].

For $\beta<0$, i.e., the absence of tachyons in $L$ eq. (2.1), we have massive gravitons of mass

$$
m_{0}=(-12 \beta)^{-1 / 2}
$$

in $L$, cf. Stelle [4]. For the weak field limit, the potential in eq. (2.7) can be simplified to be $\psi^{2} /(16 \cdot \beta)$, i.e., we have got a minimally coupled scalar field whose mass is also $m_{0}$. The superfluous factor $3 / 2$ in eq. (2.7) can be absorbed by a redefinition of $\psi$. Therefore, it is not astonishing, that all results concerning the weak field limit for both $R+R^{2}$-gravity without tachyons and Einstein gravity with a minimally coupled massive scalar field 
exactly coincide. Of course, one cannot expect this coincidence to hold for the non-linear region, too, but it is interesting to observe which properties hold there also.

We give only one example here: we consider a cosmological model of the spatially flat Friedmann type, start integrating at the quantum boundary, which is obtained by $R_{i j k l} R^{i j k l}$ on the one hand, and $T_{00}$ on the other hand, to have Planckian values, with uniformly distributed initial conditions and look whether or not an inflationary phase of the expansion appears. In both cases we get the following result: The probability $p$ to have sufficient inflation is about $p=1-\sqrt{\lambda} m_{0} / m_{\mathrm{Pl}}$, i.e., $p=99.992 \%$ if we take $m_{0}=10^{-5} m_{\mathrm{Pl}}$ from GUT and $\lambda=64$, where $e^{\lambda}$ is the linear multiplication factor of inflation.

From Quantum field theory, however, instead of the massive scalar field, a Higgs field with self-interaction turns out to he a better candidate for describing effects of the early universe. One of the advances of the latter is its possibility to describe a spontaneous breakdown of symmetry. In the following, we try to look for a purely geometric model for this Higgs field which is analogous to the above mentioned type where $L=R+R^{2}$ modelled a massive scalar field.

\subsection{The Higgs field}

For the massive scalar Field $\phi$ we have the mater Lagrangian

$$
L_{m}=-\left(\phi_{; i} \phi^{; i}-m^{2} \phi^{2}\right) / 2,
$$

and for the Higgs field to be discussed now,

$$
L_{\lambda}=-\left(\phi_{; i} \phi^{; i}+\mu^{2} \phi^{2}-\lambda \phi^{4} / 12\right) / 2 \text {. }
$$

The ground states are defined by $\phi=$ const. and $\partial L / \partial \phi=0$. This means $\phi=0$ for the scalar field, and the three ground states $\phi=\phi_{0}=0$, and

$$
\phi=\phi_{ \pm}= \pm \sqrt{6 \mu^{2} / \lambda}
$$

for the Higgs field.

The expression

$$
\left(\partial^{2} L / \partial \phi^{2}\right)^{1 / 2}
$$


represents the effective mass at these points. This gives the value $m$ for the scalar field eq. (2.9), so justifying the notation. Further, eq. (2.12) give mass $i \mu$ at $\phi=0$ and $\sqrt{2} \mu$ at $\phi=\phi_{ \pm}$for the Higgs field eq. (2.10). The imaginary value of the mass at the ground state $\phi=0$ shows the instability met there, and in the particle picture, this gives rise to a tachyon.

To have a vanishing Lagrangian at the ground state $\phi_{ \pm}$eq. (2.11) we add a constant

$$
\Lambda=-3 \mu^{4} / 2 \lambda
$$

to the Lagrangian eq. (2.10). The final Lagrangian reads

$$
L=R / 2+L_{\lambda}+\Lambda
$$

with $L_{\lambda}$ eq. (2.10) and $\Lambda$ eq. (2.13).

\subsection{The non-linear gravitational Lagrangian}

Preliminarily we direct the attention to the following fact: on the one hand, for Lagrangians (2.9), (2.10) and (2.14) the transformation $\phi \rightarrow-\phi$ is a pure gauge transformation, it does not change any invariant or geometric objects. On the other hand,

$$
R_{i j k l} \rightarrow-R_{i j k l}
$$

or simpler

$$
R \rightarrow-R
$$

is a gauge transformation at the linearized level only: taking

$$
g_{i k}=\eta_{i k}+\varepsilon h_{i k}
$$

where

$$
\eta_{i k}=\operatorname{diag}(1,-1,-1,-1),
$$

then $\varepsilon \rightarrow-\varepsilon$ implies curvature inversion eq. (2.15). To be strict: this is valid at the linearized level in $\varepsilon$ only. On the other hand, the curvature inversion eq. (2.15), and even its simpler version eq. (2.16), fails to hold quadratic in $\varepsilon$. This corresponds to fact that the $\varepsilon^{2}$-term in eq. (2.7), which corresponds 
to the $\psi^{3}$-term in the development of $\tilde{L}$ in powers of $\psi$, is the first one to break the $\psi \rightarrow-\psi$ symmetry in eq. (2.7).

In fact, the potential is essentially

$$
\left(1-e^{(-x)}\right)^{2}
$$

Calculating this, the result reads

$$
x^{2}-x^{3}+\frac{7}{12} x^{4}+\ldots
$$

Now, let us introduce the general non-linear Lagrangian $L=L(R)$ which we at the moment only assume to be an analytical function of $R$. The ground states are defined by $R=$ const., i.e.,

$$
L^{\prime} R_{i k}-g_{i k} L / 2=0 .
$$

Here, $L^{\prime}=\partial L / \partial R$.

\subsection{Calculation of the ground states}

From eq. (2.21) one immediately sees that $\partial L / \partial R=0$ defines critical values of the curvature scalar. For these values $R=R_{\text {crit }}$ it holds: For $L\left(R_{\text {crit }}\right) \neq 0$ no such ground state exists, and for $L\left(R_{\text {crit }}\right)=0$, we have only one equation $R=R_{\text {crit }}$ to be solved with 10 arbitrary functions $g_{i k}$. We call these ground states degenerated ones. For $L=R^{2}, R_{\text {crit }}=0$, this has been discussed by Buchdahl [41]. Now, let us concentrate on the case $\partial L / \partial R \neq 0$. Then $R_{i j}$ is proportional to $g_{i j}$ with a constant proportionality factor, i.e., each ground state is an Einstein space

$$
R_{i j}=R g_{i j} / 4
$$

with a prescribed constant value $R$. Inserting eq. (2.22) into eq. (2.21) we get as condition for ground states

$$
R L^{\prime}=2 L
$$

As an example, let $L$ be a third order polynomial

$$
L=\Lambda+R / 2+\beta R^{2}+\lambda R^{3} / 12 .
$$


We consider only Lagrangians with a positive linear term as we wish to reestablish Einstein gravity in the $\Lambda \rightarrow 0$ weak field limit, and $\beta<0$ to exclude tachyons there.

We now solve eq. (2.23) for the Lagrangian eq. (2.24). For $\lambda=0$ we have, independently of $\beta$, the only ground state $R=-4 \Lambda$. It is a degenerated one if and only if $\beta \Lambda=1 / 16$. That implies that for usual $R+R^{2}$ gravity eq. (2.1), i.e. $\lambda=\Lambda=0$, we get $R=0$ as the only ground state; it is a non-degenerated one.

Now, let $\lambda \neq 0$ and $\Lambda=0$. To get non-trivial ground states we need the additional assumption $\lambda>0$. Then, besides $R=0$, the ground states are

$$
R=R_{ \pm}= \pm \sqrt{6 / \lambda}
$$

being quite analogous to the ground states eq. (2.11) of the Higgs field eq. (2.10). The ground state $R=0$ is not degenerated. Of course, this statement is independent of $\lambda$ and holds true, as one knows, for $\lambda=0$. To exclude tachyons, we require $\beta<0$, then $R_{-}$is not degenerated and $R_{+}$ is degenerated if and only if $\beta=-\sqrt{6 / \lambda}$. The case $\lambda \Lambda \neq 0$ will not be considered here.

\subsection{The field equation}

For $L=L(R)$ the variation

$$
\delta(L \sqrt{-g}) / \delta g^{i j}=0
$$

gives with $L^{\prime}=\partial L / \partial R$ the following fourth order gravitational field equation

$$
L^{\prime} R_{i j}-g_{i j} L / 2+g_{i j} \square L^{\prime}-L_{; i j}^{\prime}=0
$$

It holds

$$
L_{; i j}^{\prime}=L^{\prime \prime} R_{; i j}+L^{\prime \prime \prime} R_{; i} R_{; j}
$$

With eq. (2.28), the trace of eq. (2.27) reads

$$
L^{\prime} R-2 L+3 L^{\prime \prime} \square R+3 L^{\prime \prime \prime} R_{; k} R^{; k}=0,
$$


i.e., with $L$ eq. $(2.24)$

$$
-2 \Lambda-R / 2+\lambda R^{3} / 12+6 \beta \square R+\frac{3 \lambda}{2}\left(R \square R+R_{; k} R^{; k}\right)=0 .
$$

Inserting eqs. (2.32), (2.33) and (2.28) into the 00-component of eq. (2.27) we get the equation

$$
\begin{gathered}
0=h^{2} / 2-\Lambda / 6-6 \beta\left(2 h \ddot{h}-\dot{h}^{2}+6 h^{2} \dot{h}\right) \\
+3 \lambda\left(\dot{h}+2 h^{2}\right)\left(6 h \ddot{h}+19 h^{2} \dot{h}-2 \dot{h}^{2}-2 h^{4}\right) .
\end{gathered}
$$

The remaining components are a consequence of this one.

\subsection{The Friedmann model}

Now we take as Lagrangian eq. (2.24) and as line element

$$
d s^{2}=d t^{2}-a^{2}(t)\left(d x^{2}+d y^{2}+d z^{2}\right) .
$$

The dot denotes $d / d t$ and $h=\dot{a} / a$ and we have

$$
R=-6 \dot{h}-12 h^{2} .
$$

Here we only consider the spatially flat Friedmann model eq. (2.32). Therefore, we can discuss only de Sitter stages with $R<0$, especially the ground state $R_{+}$eq. (2.25) representing an anti-de Sitter spacetime does not enter our discussion, but $R_{-}$does.

Now, let $\Lambda=0$. Solutions of eq. (2.31) with constant values $h$ are $h=0$ representing flat spacetime and in the case that $\lambda>0$ also

$$
h=\frac{1}{\sqrt[4]{24 \lambda}}
$$

representing the de Sitter spacetime. These are the non-degenerated ground states $R=0$ and $R=R_{-}=-\sqrt{6} / \lambda$, respectively. Eq. (2.31) can be written as

$$
\begin{gathered}
0=h^{2}\left(1-24 \lambda h^{4}\right) / 2+h \ddot{h}\left(1 / m_{0}^{2}+18 \lambda\left(\dot{h}+2 h^{2}\right)\right) \\
-6 \lambda \dot{h}^{3}+\dot{h}^{2}\left(45 \lambda h^{2}-1 / 2 m_{0}^{2}\right)+3 h^{2} \dot{h}\left(1 / m_{0}^{2}+36 \lambda h^{2}\right) .
\end{gathered}
$$


First, let us consider the singular curve defined by the vanishing of the coefficient of $\ddot{h}$ in eq. (2.35) in the $h-\dot{h}$-phase plane. It is, besides $h=0$, the curve

$$
\dot{h}=-2 h^{2}-1 / 18 \lambda m_{0}^{2}
$$

i.e., just the curve

$$
R=1 / 3 \lambda m_{0}^{2}=-4 \beta / \lambda
$$

which is defined by $L^{\prime \prime}=0$, cf. eq. (2.29). This value coincides with $R_{+}$if $\beta=-\sqrt{3 \lambda / 8}$, this value we need not discuss here. Points of the curve eq. (2.36) fulfil eq. (2.35) for

$$
h= \pm 1 / 18 \lambda m_{0}^{3} \sqrt{3} \sqrt{1-1 / 18 \lambda m_{0}^{4}}
$$

only, which is not real because of $\lambda \ll m_{0}^{4}$. Therefore, the space of solutions is composed of at least two connected components.

Second, for $h=0$ we have $\dot{h}=0$ or

$$
\dot{h}=-1 / 12 \lambda m^{2} .
$$

From the field equation we get: $h=\dot{h}=0$ implies $h \ddot{h} \geq 0$, i.e. $h$ does not change its sign. In a neighbourhood of eq. (2.39) we can make the ansatz

$$
h=-t / 12 \lambda m_{0}^{2}+\sum_{n=2}^{\infty} a_{n} t^{n}
$$

which has solutions with arbitrary values $a_{2}$. This means: one can change from expansion to subsequent recontraction, but only through the "eye of a needle" eq. (2.39). On the other hand, a local minimum of the scale factor never appears. Further, eq. (2.39) does not belong to the connected component of flat spacetime.

But we are especially interested in the latter one, and therefore, we restrict to the subset $\dot{h}>\dot{h}$ ( eq. (2.36)) and need only to discuss expanding solutions $h \geq 0$. Inserting $\dot{h}=0$,

$$
\ddot{h}=h\left(24 \lambda h^{4}-l\right) /\left(2 / m_{0}^{2}+72 \lambda h^{2}\right)
$$

turns out, i.e., $\ddot{h}>0$ for $h>1 / \sqrt[4]{24 \lambda}$ only. All other points in the $h-\dot{h}$ phase plane are regular ones, and one can write $d \dot{h} / d h \equiv \ddot{h} / \dot{h}=F(h, \dot{h})$ which can be calculated by eq. (2.35). 
For a concrete discussion let $\lambda \approx 10^{2} l_{\mathrm{Pl}}^{4}$ and $m_{0}=10^{-5} m_{\mathrm{Pl}}$. Then both conditions $\beta \ll-\sqrt{\lambda}$ and $\left|R_{-}\right|<l_{\mathrm{Pl}}^{-2}$ are fulfilled. Now the qualitative behaviour of the solutions can be summarized: There exist two special solutions which approximate the ground state $R_{-}$for $t \rightarrow-\infty$. All other solutions have a past singularity $h \rightarrow \infty$. Two other special solutions approximate the ground state $R_{-}$for $t \rightarrow+\infty$. Further solutions have a future singularity $h \rightarrow \infty$, and all other solutions have a power-like behaviour for $t \rightarrow \infty, a(t)$ oscillates around the classical dust model $a(t) \sim t^{2 / 3}$. But if we restrict the initial conditions to lie in a small neighbourhood of the unstable ground state $R_{-}$, only one of the following three cases appears:

1. Immediately one goes with increasing values $h$ to a singularity.

2. As a special case: one goes back to the de Sitter stage $R_{-}$.

3. The only interesting one: One starts with a finite $l_{\mathrm{Pl}^{-} \text {-valued inflationary }}$ era, goes over to a GUT-valued second inflation and ends with a power-like Friedmann behaviour.

In the last case to be considered here, let $\lambda=0, \Lambda>0$ and $\beta<0$. The analogue to eq. (2.35) then reads

$$
0=h^{2} / 2-\Lambda / 6+\left(2 h \ddot{h}-\dot{h}^{2}+6 h^{2} \dot{h}\right) / 2 m_{0}^{2} .
$$

Here, always $h \neq 0$ holds, we consider only expanding solutions $h>0$. For $\dot{h}=0$ we have

$$
\ddot{h}=\left(\Lambda m_{0}^{2} / 3-m_{0}^{2} h^{2}\right) / 2 h .
$$

For $\ddot{h}=0$ we have $\dot{h}>m_{0}^{2} / 6$ and

$$
h=\left(\Lambda / 3+\dot{h}^{2} / m_{0}^{2}\right)^{1 / 2}\left(1+6 \dot{h} / m_{0}^{2}\right)^{-1 / 2} .
$$

We obtain the following result: All solutions approach the de Sitter phase $h^{2}=\Lambda / 3$ as $t \rightarrow \infty$. There exists one special solution approaching $\dot{h}=$ $-m_{0}^{2} / 6$ for $h \rightarrow \infty$, and all solutions have a past singularity $h \rightarrow \infty$. For a sufficiently small value $\Lambda$ we have again two different inflationary eras in most of all models. 


\subsection{The generalized Bicknell theorem}

In this section we derive a general equivalence theorem between a non-linear Lagrangian $L(R)$ and a minimally coupled scalar field $\phi$ with a general potential with Einstein's theory. Instead of $\phi$ we take

$$
\psi=\sqrt{2 / 3} \phi .
$$

This is done to avoid square roots in the exponents. Then the Lagrangian for the scalar field reads

$$
\tilde{L}=\tilde{R} / 2-3 \tilde{g}^{i j} \psi_{; i} \psi_{; j} / 4+V(\psi) .
$$

At ground states $\psi=\psi_{0}$, defined by $\partial V / \partial \psi=0$ the effective mass is

$$
m=\sqrt{2 / 3} \sqrt{\partial^{2} V / \partial \psi^{2}},
$$

cf. eqs. (2.12) and (2.45). The variation $0=\delta \tilde{L} / \delta \psi$ gives

$$
0=\partial V / \partial \psi+3 \tilde{g}^{i j} \tilde{\nabla}_{i} \tilde{\nabla}_{j} \psi / 2
$$

and Einstein's equation is

$$
\tilde{E}_{i j}=\kappa \tilde{T}_{i j}
$$

with

$$
\kappa \tilde{T}_{i j}=3 \psi_{; i} \psi_{; j} / 2+\tilde{g}_{i j}\left(V(\psi)-\frac{3}{4} \tilde{g}^{a b} \psi_{; a} \psi_{; b}\right) .
$$

Now, let

$$
\tilde{g}_{i j}=e^{\psi} g_{i j} .
$$

The conformal transformation eq. (2.51) shall be inserted into eqs. (2.48), (2.49) and eq. (2.50). One obtains from eq. (2.48) with

$$
\begin{array}{r}
\psi^{; k}:=g^{i k} \psi_{; i} \\
\square \psi+\psi^{; k} \psi_{; k}=-2\left(e^{\psi} \partial V / \partial \psi\right) / 3
\end{array}
$$

and from eqs. (2.49), (2.50)

$$
E_{i j}=\psi_{; i j}+\psi_{; i} \psi_{; j}+g_{i j}\left(e^{\psi} V(\psi)-\square \psi-\psi_{; a} \psi^{; a}\right) .
$$


Its trace reads

$$
-R=4 e^{\psi} V(\psi)-3 \square \psi-3 \psi_{; a} \psi^{; a} .
$$

Comparing with eq. (2.52) one obtains

$$
R=R(\psi)=-2 e^{-\psi} \partial\left(e^{2 \psi} V(\psi)\right) / \partial \psi .
$$

Now, let us presume $\partial R / \partial \psi \neq 0$, then eq. (2.55) can be inverted as

$$
\psi=F(R) .
$$

In the last step, eq. (2.56) shall be inserted into eqs. (2.52), (2.53), (2.54). Because of

$$
F(R)_{; i j}=\partial F / \partial R \cdot R_{; i j}+\partial^{2} F / \partial R^{2} \cdot R_{; i} R_{; j}
$$

and $\partial F / \partial R \neq 0$, eq. (2.53) is a fourth-order equation for the metric $g_{i j}$. We try to find a Lagrangian $L=L(R)$ such that the equation $\delta L \sqrt{-g} / \delta g^{i j}=0$ becomes just eq. (2.53). For $L^{\prime}=\partial L / \partial R \neq 0$, eq. (2.27) can be solved to be

$$
E_{i j}=-g_{i j} R / 2+g_{i j} L / 2 L^{\prime}-g_{i j} \square L^{\prime} / L^{\prime}-L_{; i j}^{\prime} / L^{\prime} .
$$

We compare the coefficients of the $R_{; i j}$ terms in eqs. (2.53) and (2.58), this gives

$$
\begin{aligned}
\partial F / \partial R & =L^{\prime \prime} / L^{\prime}, \quad \text { hence } \\
L(R) & =\mu \int_{R_{0}}^{R} e^{F(x)} d x+\Lambda_{0}
\end{aligned}
$$

with suitable constants $\Lambda_{0}, \mu$, and $R_{0}, \mu \neq 0$. We fix them as follows: We are interested in a neighbourhood of $R=R_{0}$ and require $L^{\prime}\left(R_{0}\right)=1 / 2$. Otherwise $L$ should be multiplied by a constant factor. Further, a constant translation of $\psi$ can be used to obtain $F\left(R_{0}\right)=0$, hence $\mu=1 / 2, L\left(R_{0}\right)=$ $\Lambda_{0}$, and

$$
L^{\prime}\left(R_{0}\right)=\partial F / \partial R\left(R_{0}\right) / 2 \neq 0 .
$$

With eq. (2.59) being fulfilled, the traceless parts of eqs. (2.53) and (2.59) identically coincide. Furthermore, we have

$$
\square L^{\prime} / L^{\prime}=\square F+F^{; i} F_{; i}
$$


and it suffices to test the validity of the relation

$$
e^{F} V(F(R))=-R / 2+L / 2 L^{\prime}
$$

It holds

$$
\begin{aligned}
2 L^{\prime} & =e^{F}, \quad \text { i.e., } \\
e^{2 F} V(F(R)) & =L-R e^{F} / 2 .
\end{aligned}
$$

At $R=R_{0}$, this relation reads $V(0)=\Lambda_{0}-R_{0} / 2$. Applying $\partial / \partial R$ to eq. (2.63) gives just eq. (2.52), and, by the way, $V^{\prime}(0)=R_{0} / 2-2 \Lambda_{0}$. In sum,

$$
L(R)=V(0)+R_{0} / 2+\int_{R_{0}}^{R} e^{F(x)} d x / 2
$$

where $F(x)$ is defined via $F\left(R_{0}\right)=0$,

$$
\psi=F\left(-2 e^{-\psi} \partial\left(e^{2 \psi} V(\psi)\right) / \partial \psi\right)
$$

Now, let us go the other direction: Let $L=L(R)$ be given such that at $R=R_{0}, L^{\prime} L^{\prime \prime} \neq 0$. By a constant change of $L$ let $L^{\prime}\left(R_{0}\right)=1 / 2$. Define $\Lambda_{0}=L\left(R_{0}\right), \psi=F(R)=\ln \left(2 L^{\prime}(R)\right)$ and consider the inverted function $R=F^{-1}(\psi)$. Then

$$
V(\psi)=\left(\Lambda_{0}-R_{0} / 2\right) e^{-2 \psi}-e^{-2 \psi} \int_{0}^{\psi} e^{x} F^{-1}(x) d x / 2
$$

is the potential ensuring the above mentioned conformal equivalence. This procedure is possible at all $R$-intervals where $L^{\prime} L^{\prime \prime} \neq 0$ holds. For analytical functions $L(R)$, this inequality can be violated for discrete values $R$ only, or one has simply a linear function $L(R)$ being Einstein gravity with $\Lambda$-term.

It turned out that this integral eq. (2.66) can be evaluated in closed form as follows:

$$
V(\psi)=L\left(F^{-1}(\psi)\right) \cdot e^{-2 \psi}-\frac{1}{2} F^{-1}(\psi) \cdot e^{-\psi} .
$$

Examples: 1 . Let $L=\Lambda+R^{2}, R_{0}=1 / 4$, then $4 R=e^{\psi}$ and

$$
V(\psi)=\Lambda e^{-2 \psi}-1 / 16
$$


2. Let $L=\Lambda+R / 2+\beta R^{2}+\lambda R^{3} / 12, \quad R_{0}=0$, hence $\beta \neq 0$ is necessary. We get

$$
\begin{array}{r}
e^{\psi}-1=4 \beta R+\lambda R^{2} / 2 \quad \text { and } \\
V(\psi)=\Lambda e^{-2 \psi}+ \\
2 \beta \lambda^{-1} e^{-2 \psi}\left(e^{\psi}-1-16 \beta^{2}(3 \lambda)^{-1}\left(\left(1+\lambda\left(e^{\psi}-l\right) / 8 \beta^{2}\right)^{3 / 2}-1\right)\right) .
\end{array}
$$

The limit $\lambda \rightarrow 0$ in eq. (2.69) is possible and leads to

$$
V(\psi)=\Lambda e^{-2 \psi}-\left(1-e^{-\psi}\right)^{2} / 16 \beta
$$

so we get for $\Lambda=0$ again the potential from eq. (2.7).

Now, let $R_{0}$ be a non-degenerated ground state, hence

$$
L(R)=\Lambda_{0}+\left(R-R_{0}\right) / 2+L^{\prime \prime}\left(R_{0}\right)\left(R-R_{0}\right)^{2} / 2+\ldots
$$

with $L^{\prime \prime}\left(R_{0}\right) \neq 0$ and $\Lambda_{0}=R_{0} / 4$, cf. subsection 2.4. Using eq. (2.66) we get $V^{\prime}(0)=0$ and

$$
V^{\prime \prime}(0)=R_{0} / 2-1 /\left(4 L^{\prime \prime}\left(R_{0}\right)\right)
$$

\subsection{The generalized equivalence}

It is worth mentioning that this conformal equivalence theorem can be formulated for arbitrary dimensions $n>2$ : Let

$$
\tilde{\mathcal{L}}=\tilde{R} / 2-\frac{1}{2} \tilde{g}^{i j} \phi_{\mid i} \phi_{\mid j}+V(\phi)
$$

and

$$
\tilde{g}_{i j}=e^{\lambda \phi} g_{i j}, \quad \lambda=\frac{2}{(n-1)(n-2)}
$$

be the conformally transformed metric. Then the solutions of the variation

of eq. (2.73) are transformed by eq. (2.74) to the solutions of the variation of $L=L(R)$, where

$$
R=-2 e^{\lambda \phi}\left(\frac{n V}{n-2}+\mu \frac{d V}{d \phi}\right), \quad \mu=\sqrt{\frac{n-1}{n-2}}
$$


is supposed to be locally, i.e. near $R=R_{0}$, invertible as

$$
\begin{array}{r}
\phi=F(R), \quad F\left(R_{0}\right)=0, \quad F^{\prime}\left(R_{0}\right) \neq 0 \\
\mathcal{L}(R)=\frac{1}{2} R_{0}+V(0)+\frac{1}{2} \int_{R_{0}}^{R} e^{F(x) / \mu} d x .
\end{array}
$$

The inverse direction is possible provided $L^{\prime}(R) \cdot L^{\prime \prime}(R) \neq 0$.

\subsection{The fourth order gravity model}

Now we come to the following question: Let $L(R)$ be a $C^{3}$-function fulfilling $L(0)=0, L^{\prime}(0) \cdot L^{\prime \prime}(0)<0$. Then we can write

$$
\mathcal{L}(R)=\frac{R}{2}+\beta R^{2}+O\left(R^{3}\right), \quad \beta<0 .
$$

We consider the Bianchi-type I vacuum solutions which start in a neighbourhood of the Minkowski spacetime and ask for the behaviour as $t \rightarrow \infty$. Applying the equivalence theorem we arrive at the models discussed before, and this is applicable for $|R|$ being small enough. The conformal factor depends on $t$ only, and therefore, the space of Bianchi-type I models will not be leaved, and we can formulate the following: In a neighbourhood of Minkowski spacetime, all Bianchi-type I models which represent a stationary point of the action eq. (2.77), can be integrated up to $t \rightarrow \infty$ or $-\infty$, let it be $+\infty$. One singular solution is the Kasner solution and all other solutions undergo isotropization and have an averaged equation of state $p=0$ for $t \rightarrow \infty$.

The de Sitter spacetime

$$
d s^{2}=d t^{2}-e^{2 H t}\left(d x^{2}+d y^{2}+d z^{2}\right), \quad H \neq 0
$$

is the spacetime being mainly discussed to represent the inflationary phase of cosmic evolution. However, a spacetime defined by

$$
d s^{2}=d t^{2}-|t|^{2 p}\left(d x^{2}+d y^{2}+d z^{2}\right), \quad p \neq 0
$$

enjoys increasing interest for these discussions, too. Especially, eq. (2.79) with $p \geq 1, t>0$ is called power-law inflation; and with $p<0, t<0$ it is called polar inflation. 
Eq. (2.79) defines a self-similar spacetime: if we multiply the metric $d s^{2}$ by an arbitrary positive constant $a^{2}$, then the resulting $d \hat{s}^{2}=a^{2} d s^{2}$ is isometric to $d s^{2}$. Proof: We perform a coordinate transformation $\hat{t}=a t$, $\hat{x}=b(a, p) x \ldots$ On the other hand, the de Sitter spacetime eq. (2.78) is not self-similar, because it has a constant non-vanishing curvature scalar.

Power-law inflation is intrinsically time-oriented. Proof: The gradient of the curvature scalar defines a temporal orientation. On the other hand, the expanding $(H>0)$ and the contracting $(H<0)$ de Sitter spacetime can be transformed into each other by a coordinate transformation, because both of them can be transformed to the closed Friedmann universe with scale factor $\cosh (H t)$, which is an even function of $t$. This property is connected with the fact that eq. (2.79) gives a global description, whereas eq. (2.78) gives only a proper subset of the full de Sitter spacetime.

For $p \rightarrow \infty$, eq. (2.79) tends to eq. (2.78). Such a statement has to be taken with care, even for the case with real functions. Even more carefully one has to deal with spacetimes. The most often used limit - the Gerochlimit of spacetimes - has the property that a symmetry, here we take it to be self-similarity, of all the elements of the sequence must also be a symmetry of the limit.

From this it follows that the Geroch limit of spacetimes eq. (2.79) with $p \rightarrow \infty$ cannot be unique, moreover, it is just the one-parameter set eq. (2.78) parametrized by arbitrary values $H>0$.

\section{Cosmic no hair theorem and Newtonian limit}

We now discuss the cosmic no hair theorem, which tells under which circumstances the de Sitter spacetime represents an attractor solution within the set of other nearby solutions. This property ensures the inflationary model to be a typical solution. The notion "cosmic no hair theorem" is chosen because of its analogous properties to the "no hair theorem" for black holes.

After a general introduction we restrict our consideration to spatially flat 
Friedmann models. In this section, we choose gravitational Lagrangians

$$
R \square^{k} R \sqrt{-g}
$$

and linear combinations of them. They are motivated from trials how to overcome the non-renormalizability [3, 4] of Einstein's theory of gravity. Results are: For arbitrary $k$, i.e., for arbitrarily large order $2 k+4$ of the field equation, one can always find examples where the attractor property takes place. Such examples necessarily need a non-vanishing $R^{2}$-term. The main formulas do not depend on the dimension, so one gets similar results also for 1+1-dimensional gravity and for Kaluza-Klein cosmology.

Over the years, the notion "no hair conjecture" drifted to "no hair theorem" without possessing a generally accepted formulation or even a complete proof. Several trials have been made to formulate and prove it at least for certain special cases. They all have the overall structure: "For a geometrically defined class of spacetimes and physically motivated properties of the energy-momentum tensor, all the solutions of the gravitational field equation asymptotically converge to a space of constant curvature."

Weyl wrote: "The behaviour of every world satisfying certain natural homogeneity conditions in the large follows the de Sitter solution asymptotically." Barrow and Götz apply the formulation: "All ever-expanding universes with $\Lambda>0$ approach the de Sitter spacetime locally."

Barrow gave examples that the no hair conjecture fails if the energy condition is relaxed and pointed out, that this is necessary to solve the graceful exit problem. He uses the formulation of the no hair conjecture "in the presence of an effective cosmological constant, stemming e.g. from viscosity, the de Sitter spacetime is a stable asymptotic solution".

In the three papers [80], Prigogine et al. developed a phenomenological model of particle and entropy creation. It allows particle creation from spacetime curvature, but the inverse procedure, i.e. particle decay into spacetime curvature is forbidden. This breaks the $t \longrightarrow-t$-invariance of the model. Within that model, the expanding de Sitter spacetime is an attractor solution independently of the initial fluctuations; this means, only the expanding de Sitter solution is thermodynamically possible.

Vilenkin discussed future-eternal inflating universe models: they must 
have a singularity if the condition D: "There is at least one point $p$ such that for some point $q$ to the future of $p$ the volume of the difference of the pasts of $p$ and $q$ is finite" is fulfilled.

In Maeda [77] the following argument is given: If the matter distribution is too clumpy, then a large number of small black holes appears. Then one should look for an inflationary scenario where these black holes are harmless. They cannot clump together to one giant black hole because of the exponential expansion of the universe; this explains the existing upper bound of the mass of black holes in the quasi-de Sitter model: above

$$
M_{\text {crit }}=\frac{1}{3 \sqrt{\Lambda}}
$$

there do not exist horizons; this restriction is called cosmic hoop conjecture.

For the no hair conjecture for $R^{2}$ models one uses the formulation "asymptotical de Sitter, at least on patch". The restriction "on patch" is not strictly defined but refers to a kind of local validity of the statement, e.g., in a region being covered by one single synchronized system of reference in which the spatial curvature is non-positive and the energy conditions are fulfilled. The Starobinsky model [103] is one of those which does not need an additional inflaton field to get the desired quasi de Sitter stage, see $[100,101,104]$ as three of the earliest approaches to this point.

A further result is that by the addition of a cosmological term, the Starobinsky model leads naturally to double inflation. Let us comment this result: It is correct, but one should add that this is got at the price of getting a "graceful exit problem", by this phrase there is meant the problem of how to finish the inflationary phase dynamically - in the Starobinsky model this problem is automatically solved by the fact that the quasi de Sitter phase is a transient attractor only.

The paper Buchdahl [38] deals with Lagrangians of arbitrarily high order. Its results are applied in $[63,65,81]$ to general Lagrangians $F(R, \square)$. The original idea of A. D. Sakharov [54] from 1967 was to define higher order curvature corrections to the Einstein action to get a kind of elasticity of the vacuum. Then the usual breakdown of measurements at the Planck length, such a short de Broglie wave length corresponds to such a large mass which makes the measuring apparatus to a black hole, is softened. 


\subsection{Definition of an asymptotic de Sitter spacetime}

In this subsection we want to compare some possible definitions of an asymptotic de Sitter spacetime. To this end let us consider the metric

$$
d s^{2}=d t^{2}-e^{2 \alpha(t)} \sum_{i=1}^{n} d\left(x^{i}\right)^{2}
$$

which is the spatially flat Friedmann model in $n$ spatial dimensions. We consider all values $n \geq 1$, but then concentrate on the usual case $n=3$. The Hubble parameter is

$$
H=\dot{\alpha} \equiv \frac{d \alpha}{d t}
$$

We get

$$
R_{00}=-n\left(\frac{d H}{d t}+H^{2}\right), \quad R=-2 n \dot{H}-n(n+1) H^{2} .
$$

Then it holds: The following 4 conditions for metric (3.3) are equivalent. 1: The spacetime is flat. 2: It holds $R=R_{00}=0$. 3: The curvature invariant $R_{i j} R^{i j}$ vanishes. 4: Either $\alpha=$ const. or

$$
n=1 \quad \text { and } \quad \alpha=\ln \left|t-t_{0}\right|+\text { const. }
$$

For the last case with $n=1$ one has to observe that

$$
d s^{2}=d t^{2}-\left(t-t_{0}\right)^{2} d x^{2}
$$

represents flat spacetime in polar coordinates. For the proof we use the identity

$$
R_{i j} R^{i j}=\left(R_{00}\right)^{2}+\frac{1}{n}\left(R-R_{00}\right)^{2}
$$

An analogous characterization is valid for the de Sitter spacetime. The following 4 conditions for metric (3.3) are equivalent. 1: It is a non-flat spacetime of constant curvature. $2: R_{00}=R /(n+1)=$ const. $\neq 0 . \quad 3$ : $(n+1) R_{i j} R^{i j}=R^{2}=$ const. $\neq 0$. 4: Either $H=$ const. $\neq 0$ or

$$
n=1 \text { and }\left(d s^{2}=d t^{2}-\sin ^{2}(\lambda t) d x^{2} \text { or } d s^{2}=d t^{2}-\sinh ^{2}(\lambda t) d x^{2}\right) .
$$


For $n=1$, the de Sitter spacetime and anti-de Sitter spacetime differ by the factor -1 in front of the metric only. For $n>1$, only the de Sitter spacetime, having $R<0$, is covered, because the anti-de Sitter spacetime cannot be represented as spatially flat Friedmann model. Our result shows that within the class of spatially flat Friedmann models, a characterization of the de Sitter spacetime using polynomial curvature invariants only, is possible.

Next, let us look for isometries leaving the form of the metric (3.3) invariant. Besides spatial isometries, the map $\alpha \rightarrow \tilde{\alpha}$ defined by

$$
\tilde{\alpha}(t)=c+\alpha\left( \pm t+t_{0}\right), \quad c, t_{0}=\text { const. }
$$

leads to an isometric spacetime. The simplest expressions being invariant by such a transformation are $H^{2}$ and $\dot{H}$. We take $\alpha$ as dimensionless, then $H$ is an inverse time and $\dot{H}$ an inverse time squared. Let $H \neq 0$ in the following. The expression

$$
\varepsilon:=\dot{H} H^{-2}
$$

is the simplest dimensionless quantity defined for the spatially flat Friedmann models and being invariant with respect to this transformation. Let $n>1$ in the following, then it holds: Two metrics of type (3.3) are isometric if and only if the corresponding functions $\alpha$ and $\tilde{\alpha}$ are related by this transformation. It follows: Metric (3.3) with $H \neq 0$ represents the de Sitter spacetime if and only if $\varepsilon \equiv 0$.

All dimensionless invariants containing at most second order derivatives of the metric can be expressed as $f(\varepsilon)$, where $f$ is any given function. But if one has no restriction to the order, one gets a sequence of further invariants

$$
\varepsilon_{2}=\ddot{H} H^{-3}, \quad \ldots, \varepsilon_{p}=\frac{d^{p} H}{d t^{p}} H^{-p-1} .
$$

Let $H>0$ in metric (3.3) with $n>1$. We call it an asymptotic de Sitter spacetime if

$$
\lim _{t \rightarrow \infty} \frac{\alpha(t)}{t}=\text { const. } \neq 0
$$

or

$$
\lim _{t \rightarrow \infty} R^{2}=\text { const. }>0 \quad \text { and } \quad \lim _{t \rightarrow \infty}(n+1) R_{i j} R i j-R^{2}=0
$$


or for some natural number $p$ one has

$$
\lim _{t \rightarrow \infty} \varepsilon_{j}=0 \quad \text { for } \quad 1 \leq j \leq p .
$$

In general, all these definitions are different. Using the identity

$$
R_{i j} R^{i j}=n^{2}\left(\dot{H}+H^{2}\right)^{2}+n\left(\dot{H}+n H^{2}\right)^{2}
$$

we will see that all these definitions lead to the same result if we restrict ourselves to the set of solutions of the higher-order field equations. The problem is that none of the above definitions can be generalized to inhomogeneous models. One should find a polynomial curvature invariant which equals a positive constant if and only if the spacetime is locally the de Sitter spacetime. To our knowledge, such an invariant cannot be found in the literature, but also the non-existence of such an invariant has not been proven up to now.

This situation is quite different for the positive definite case: For signature $(++++)$ and $S_{i j}=R_{i j}-\frac{R}{4} g_{i j}$ it holds:

$$
I \equiv\left(R-R_{0}\right)^{2}+C_{i j k l} C^{i j k l}+S_{i j} S^{i j}=0
$$

if and only if the $V_{4}$ is a space of constant curvature $R_{0}$. So $I \longrightarrow 0$ is a suitable definition of an asymptotic space of constant curvature. One possibility exists, however, for the Lorentz signature case, if one allows additional structure as follows: An ideal fluid has an energy-momentum tensor

$$
T_{i j}=(\rho+p) u_{i} u_{j}-p g_{i j}
$$

where $u_{i}$ is a continuous vector field with $u_{i} u^{i} \equiv 1$. For matter with equation of state $\rho=-p$, the equation $T_{; j}^{i j} \equiv 0$ implies $p=$ const., and so every solution of Einstein's theory with such matter is isometric to a vacuum solution of Einstein's theory with a cosmological term. The inverse statement, however, is valid only locally:

Given a vacuum solution of Einstein's theory with a $\Lambda$-term, one has to find continuous timelike unit vector fields which need not to exist from topological reasons. And if they exist, they are not at all unique. So, it becomes possible to define an invariant $J$ which vanishes if and only if the 
spacetime is de Sitter by transvecting the curvature tensor with $u^{i} u^{j}$ and/or

$g^{i j}$ and suitable linear and quadratic combinations of such terms. Then time $t$ becomes defined by the streamlines of the vector $u^{i}$. If one defines the asymptotic de Sitter spacetime by $J \longrightarrow 0$ as $t \longrightarrow \infty$, then it turns out, that this definition is not independent of the vector field $u^{i}$.

\subsection{Lagrangian $F\left(R, \square R, \square^{2} R, \ldots, \square^{k} R\right)$}

Let us consider the Lagrangian density $L$ given by

$$
L=F\left(R, \square R, \square^{2} R, \ldots, \square^{k} R\right) \sqrt{-g}
$$

where $R$ is the curvature scalar, $\square$ the D'Alembertian and $g_{i j}$ the metric of a Pseudo-Riemannian $V_{D}$ of dimension $D \geq 2$ and arbitrary signature; $g=-\left|\operatorname{det} g_{i j}\right|$. The main application will be $D=4$ and metric signature $(+---) . F$ is supposed to be a sufficiently smooth function of its arguments, preferably a polynomial. Buchdahl already dealt with such kind of Lagrangians in 1951, but then it became quiet of them for decades.

The variational derivative of $L$ with respect to the metric yields the tensor

$$
P^{i j}=-\frac{1}{\sqrt{-g}} \frac{\delta L}{\delta g_{i j}}
$$

The components of this tensor read

$$
P_{i j}=G R_{i j}-\frac{1}{2} F g_{i j}-G_{; i j}+g_{i j} \square G+X_{i j}
$$

where the semicolon denotes the covariant derivative, $R_{i j}$ the Ricci tensor, and

$$
X_{i j}=\sum_{A=1}^{k} \frac{1}{2} g_{i j}\left[F_{A}\left(\square^{A-1} R\right)^{; m}\right]_{; m}-F_{A(; i}\left[\square^{A-1} R\right]_{; j)}
$$

having the round symmetrization brackets in its last term. For $k=0$, i.e. $F=F(R)$, a case considered in subsection 3.3, the tensor $X_{i j}$ identically vanishes. It remains to define the expressions $F_{A}, A=0, \ldots, k$.

$$
F_{k}=\frac{\partial F}{\partial \square^{k} R}
$$


and for $A=k-1, \ldots, 0$

$$
F_{A}=\square F_{A+1}+\frac{\partial F}{\partial \square^{A} R}
$$

and finally $G=F_{0}$. The brackets are essential, for any scalar $\Phi$ it holds

$$
\square\left(\Phi_{; i}\right)-(\square \Phi)_{; i}=R_{i}{ }^{j} \Phi_{; j}
$$

Inserting $\Phi=\square^{m} R$ into this equation, one gets identities to be applied in the sequel without further notice. The covariant form of energy-momentum conservation reads

$$
P_{j ; i}^{i} \equiv 0
$$

and $P_{i j}$ identically vanishes if and only if $F$ is a divergence, i.e., locally there can be found a vector $v^{i}$ such that $F=v_{; i}^{i}$ holds. Remark: Even for compact manifolds without boundary the restriction "locally" is unavoidable, for example, let $D=2$ and $V_{2}$ be the Riemannian two-sphere $S^{2}$ with arbitrary positive definite metric. $R$ is a divergence, but there do not exist continuous vector fields $v^{i}$ fulfilling $R=v_{; i}^{i}$ on the whole $S^{2}$. Example: for $m, n \geq 0$ it holds

$$
\square^{m} R \square^{n} R-R \square^{m+n} R=\text { divergence. }
$$

So, the terms $\square^{m} R \square^{n} R$ with naturals $m$ and $n$ can be restricted to the case $m=0$ without loss of generality.

\subsection{No hair theorems for higher-order gravity}

For $n>1$, the $n+1$-dimensional de Sitter spacetime is an attractor solution for the field equation derived from the Lagrangian

$$
R^{(n+1) / 2} .
$$

It is not an attractor solution for the Lagrangian $R \square^{k} R$ and $k>0$. There exist combinations of coefficients $c_{i}$, such that the de Sitter spacetime is an attractor solution for the field equation derived from the more general Lagrangian

$$
L=c_{0} R^{(n+1) / 2}+\sum_{k=0}^{m} c_{k} R \square^{k} R .
$$


Idea of Proof: Concerning fourth-order gravity this method was previously applied e.g. by Barrow [64]. The de Sitter spacetime is an exact solution for the field equation, if and only if $2 R G=(n+1) F$. If we make the ansatz

$$
\dot{\alpha}(t)=1+\beta(t)
$$

we get the linearized field equation $0=\ddot{\beta}+n \dot{\beta}$ for the Lagrangian $R^{\frac{n+1}{2}}$. For the Lagrangian $L=R \square^{k} R$ we get the linearized field equation $\square^{k} R=$ $\left(\square^{k} R\right)_{, 0}$. For the characteristic polynomial we get a recursive formula such that the next order is received from the previous one by multiplying with $\cdot x \cdot(x+n)$. We get the roots $x_{1}=-n-1, x_{2}=-n(k$-fold $), x_{3}=0$ ( $k$-fold) and $x_{4}=+1$. Because of the last root the de Sitter spacetime is not an attractor solution. For the Lagrangian (3.29) we get the characteristic polynomial

$$
P(x)=x(x+n)\left[c_{0}+\sum_{k=1}^{m} c_{k} x^{k-1}(x+n)^{k-1}(x-1)(x+n+1)\right]
$$

for the linearized field equation. The transformation $z=x^{2}+n x+\frac{n^{2}}{4}$ gives a polynomial $Q(z)$ which can be solved explicitly. So one can find those combinations of the coefficients such that the de Sitter spacetime is an attractor solution.

It turned out that all the variants of the definition of an asymptotic de Sitter solution given in subsection 3.1 lead to the same class of solutions, i.e., the validity of the theorems written below does not depend on which of the variants of definition of an asymptotic de Sitter spacetime listed is applied. For the 6th-order case we can summarize as follows: Let

$$
L=R^{2}+c_{1} R \square R
$$

and

$$
L_{\mathrm{E}}=R-\frac{l^{2}}{6} L
$$

with length $l>0$. Then the following statements are equivalent.

1. The Newtonian limit of $L_{\mathrm{E}}$ is well-behaved, and the potential $\phi$ consists of terms $e^{-\alpha r} / r$ with $\alpha \geq 0$ only. 
2. The de Sitter spacetime with $H=1 / l$ is an attractor solution for $L$ in the set of spatially flat Friedmann models, and this can already be seen from the linearized field equation.

3. The coefficient $c_{1} \geq 0$, and the graceful exit problem is solved for the quasi de Sitter phase $H \leq 1 / l$ of $L_{\mathrm{E}}$.

4. $l^{2}=l_{0}^{2}+l_{1}^{2}$ such that $l^{2} c_{1}=l_{0}^{2} l_{1}^{2}$ has a solution with $0 \leq l_{0}<l_{1}$.

5. $0 \leq c_{1}<l^{2} / 4$.

A formulation which includes also the marginally well-behaved cases reads as follows: Let $L$ and $L_{\mathrm{E}}$ as in the previous result, then the following statements are equivalent:

1. The Newtonian limit of $L_{\mathrm{E}}$ is well-behaved, for the potential $\phi$ we allow $1 / r$ and terms like

$$
\frac{P(r)}{r} e^{-\alpha r} \quad \text { with } \quad \alpha>0
$$

and a polynomial $P$.

2. The de Sitter spacetime with $H=1 / l$ cannot be ruled out to be an attractor solution for $L$ in the set of spatially flat Friedmann models if one considers the linearized field equation only.

3. $L_{\mathrm{E}}$ is tachyonic-free.

4. $l^{2}=l_{0}^{2}+l_{1}^{2}$ with $l^{2} c_{1}=l_{0}^{2} l_{1}^{2}$ has a solution with $0 \leq l_{0} \leq l_{1}$.

5. For the coefficients we have $0 \leq c_{1} \leq \frac{l^{2}}{4}$.

\subsection{Discussion of no hair theorems}

In subsection 3.3 we have shown: The results of the Starobinsky model are structurally stable with respect to the addition of a sixth-order term $\sim R \square R$, if the coefficients fufil certain inequalities. For the eighth-order case we got: For

$$
L=R^{2}+c_{1} R \square R+c_{2} R \square \square R, \quad c_{2} \neq 0
$$

and the case $n>1$ the de Sitter spacetime with $H=1$ is an attractor solution in the set of spatially flat Friedmann models if and only if the following inequalities are fulfilled:

$$
0<c_{1}<\frac{1}{n+1}, \quad 0<c_{2}<\frac{1}{(n+1)^{2}}
$$


and

$$
c_{1}>-\left(n^{2}+n+1\right) c_{2}+\sqrt{\left(n^{4}+4 n^{3}+4 n^{2}\right) c_{2}^{2}+4 c_{2}}
$$

These inequalities define an open region in the $c_{1}-c_{2}$-plane whose boundary contains the origin; and for the other boundary points the linearized equation does not suffice to decide the attractor property.

This situation shall be called "semi-attractor" for simplicity. In a general context this notion is used to describe a situation where all Lyapunov coefficients have non-positive real parts and at least one of them is purely imaginary.

In contrary to the 6th-order case, here we do not have a one-to-one correspondence, but a non-void open intersection with that parameter set having the Newtonian limit for $L_{E}$ well-behaved.

To find out, whether another de Sitter spacetime with an arbitrary Hubble parameter $H>0$ is an attractor solution for the eighth-order field equation following from the above Lagrangian, one should remember that $H$ has the physical dimension of an inverted time, $c_{1}$ is a time squared, $c_{2}$ is a time to power 4 . So, we have to replace $c_{1}$ by $c_{1} H^{2}$ and $c_{2}$ by $c_{2} H^{4}$ in the above dimensionless inequalities to get the correct conditions. Example:

$$
0<c_{1} H^{2}<\frac{1}{4}
$$

Let us summarize: Here for a theory of gravity of order higher than four the Newtonian limit and the attractor property of the de Sitter spacetime are systematically compared. It should be noted that the details of the theory sensibly depend on the numerical values of the corresponding coefficients. So, no general overall result about this class of theories is ever to be expected.

We have found out that for the class of theories considered here, one of the typical indicators of instability - cosmological runaway-solutions - need not to exist, even for an arbitrarily high order of the field equation. It is an additional satisfactory result that this takes place in the same range of parameters where the Newtonian limit is well behaved. 


\subsection{The Newtonian limit of 4th-order gravity}

Let us consider the gravitational theory defined by the Lagrangian

$$
L_{\mathrm{g}}=(8 \pi G)^{-1}\left(R / 2+\left(\alpha R_{i j} R^{i j}+\beta R^{2}\right) l^{2}\right) .
$$

$G$ is Newton's constant, $l$ a coupling length and $\alpha$ and $\beta$ numerical parameters. $R_{i j}$ and $R$ are the Ricci tensor and its trace. Introducing the matter Lagrangian $L_{\mathrm{m}}$ and varying $L_{\mathrm{g}}+L_{\mathrm{m}}$ one obtains the field equation

$$
E_{i j}+\alpha H_{i j}+\beta G_{i j}=8 \pi G T_{i j} .
$$

For $\alpha=\beta=0$ this reduces to General Relativity Theory. The explicit expressions $H_{i j}$ and $G_{i j}$ can be found in Stelle [59].

In a well-defined sense, the weak-field slow-motion limit of Einstein's theory is just Newton's theory. In the following, we consider the analogous problem for fourth-order gravity eqs. (3.38), (3.39).

The slow-motion limit can be equivalently described as the limit $c \rightarrow \infty$, where $c$ is the velocity of light. In this sense we have to take the limit $G \rightarrow 0$ while $G \cdot c$ and $l$ remain constants. Then the energy-momentum tensor $T_{i j}$ reduces to the rest mass density $\rho$ :

$$
T_{i j}=\delta_{i}^{0} \delta_{j}^{0} \rho,
$$

$x^{0}=t$ being the time coordinate. The metric can be written as

$$
d s^{2}=(1-2 \phi) d t^{2}-(1+2 \psi)\left(d x^{2}+d y^{2}+d z^{2}\right) .
$$

Now eqs. (3.40) and (3.41) will be inserted into eq. (3.39). In our approach, products and time derivatives of $\phi$ and $\psi$ can be neglected, i.e.,

$$
R=4 \Delta \psi-2 \Delta \phi, \quad \text { where } \quad \Delta f=f_{, x x}+f_{, y y}+f_{, z z} .
$$

Further $R_{00}=-\Delta \phi, H_{00}=-2 \Delta R_{00}-\Delta R$ and $G_{00}=-4 \Delta R$, where $l=1$.

Then it holds: The validity of the 00-component and of the trace of eq. (3.39),

$$
R_{00}-R / 2+\alpha H_{00}+\beta G_{00}=8 \pi G \rho
$$


and

$$
-R-4(\alpha+3 \beta) \Delta R=8 \pi G \rho,
$$

imply the validity of the full eq. (3.39).

Now, let us discuss eqs. (3.43) and (3.44) in more details: Eq. (3.43) reads

$$
-\Delta \phi-R / 2+\alpha(2 \Delta \Delta \phi-\Delta R)-4 \beta \Delta R=8 \pi G \rho .
$$

Subtracting one half of eq. (3.44) yields

$$
-\Delta \phi+2 \alpha \Delta \Delta \phi+(\alpha+2 \beta) \Delta R=4 \pi G \rho .
$$

For $\alpha+2 \beta=0$ one obtains

$$
-(1-2 \alpha \Delta) \Delta \phi=4 \pi G \rho
$$

and then $\psi=\phi$ is a solution of eqs. (3.43), (3.44). For all other cases the equations for $\phi$ and $\psi$ do not decouple immediately, but, to get equations comparable with Poisson's equation we apply $\Delta$ to eq. (3.44) and continue as follows.

For $\alpha+3 \beta=0$ one gets from eq. (3.46)

$$
-(1-2 \alpha \Delta) \Delta \phi=4 \pi G(1+2 \alpha \Delta / 3) \rho .
$$

The $\Delta$-operator applied to the source term in eq. (3.48) is only due to the application of $\Delta$ to the trace, the original equations (3.43), (3.44) contain only $\rho$ itself.

For $\alpha=0$ one obtains similarly the equation

$$
-(1+12 \beta \Delta) \Delta \phi=4 \pi G(1+16 \beta \Delta) \rho .
$$

For all other cases - just the cases not yet covered by the literature - the elimination of $\psi$ from the system (3.43), (3.44) gives rise to a sixth-order equation

$$
-(1+4(\alpha+3 \beta) \Delta)(1-2 \alpha \Delta) \Delta \phi=4 \pi G(1+2(3 \alpha+8 \beta) \Delta) \rho .
$$

Fourth order gravity is motivated by quantum-gravity considerations and therefore, its long-range behaviour should be the same as in Newton's theory. 
Therefore, the signs of the parameters $\alpha, \beta$ should be chosen to guarantee an exponentially vanishing and not an oscillating behaviour of the fourth-order terms:

$$
\alpha \geq 0, \quad \alpha+3 \beta \leq 0 .
$$

On the other hand, comparing parts of eq. (3.50) with the Proca equation it makes sense to define the masses

$$
m_{2}=(2 \alpha)^{-1 / 2} \quad \text { and } \quad m_{0}=(-4(\alpha+3 \beta))^{-1 / 2} .
$$

Then eq. (3.51) prevents the masses of the spin 2 and spin 0 gravitons to become imaginary.

Now, inserting a delta source $\rho=m \delta$ into eq. (3.50) one obtains for $\phi$ the same result as Stelle [59],

$$
\phi=m r^{-1}\left(1+\exp \left(-m_{0} r\right) / 3-4 \exp \left(-m_{2} r\right) / 3\right) .
$$

To obtain the metric completely one has also to calculate $\psi$. It reads

$$
\psi=m r^{-1}\left(1-\exp \left(-m_{0} r\right) / 3-2 \exp \left(-m_{2} r\right) / 3\right)
$$

For finite values $m_{0}$ and $m_{2}$ these are both bounded functions, also for $r \rightarrow 0$. In the limits $\alpha \rightarrow 0$, i.e. $m_{2} \rightarrow \infty$, and $\alpha+3 \beta \rightarrow 0$, i.e. $m_{0} \rightarrow \infty$, the terms with $m_{0}$ and $m_{2}$ in eqs. (3.53) and (3.54) simply vanish. For these cases $\phi$ and $\psi$ become unbounded as $r \rightarrow 0$.

Inserting eqs. (3.53) and (3.54) into the metric (3.41), the behaviour of the geodesics shall be studied. First, for an estimation of the sign of the gravitational force we take a test particle at rest and look whether it starts falling towards the centre or not. The result is: for $m_{0} \leq 2 m_{2}$, gravitation is always attractive, and for $m_{0}>2 m_{2}$ it is attractive for large but repelling for small distances. The intermediate case $m_{0}=2 m_{2}$, i.e., $3 \alpha+8 \beta=0$, is already known to be a special one from eq. (3.50).

Next, let us study the perihelion advance for distorted circle-like orbits. Besides the general relativistic perihelion advance, which vanishes in the Newtonian limit, we have an additional one of the following behaviour: for $r \rightarrow 0$ and $r \rightarrow \infty$ it vanishes and for $r \approx 1 / m_{0}$ and $r \approx 1 / m_{2}$ it has local maxima, i.e., resonances. 
Finally, it should be mentioned that the gravitational field of an extended body can be obtained by integrating eqs. (3.53) and (3.54). For a spherically symmetric body the far field is also of the type

$$
m r^{-1}\left(1+a \exp \left(-m_{0} r\right)+b \exp \left(-m_{2} r\right)\right)
$$

and the factors $a$ and $b$ carry information about the mass distribution inside the body.

\subsection{Higher-order gravity}

One-loop quantum corrections to the Einstein equation can be described by curvature-squared terms and lead to fourth order gravitational field equations; their Newtonian limit is described by a potential "Newton + one Yukawa term". A Yukawa potential has the form $\exp (-r / l) / r$ and was originally used by Yukawa to describe the meson field.

Higher-loop quantum corrections to the Einstein equation are expected to contain terms of the type $R \square^{k} R$ in the Lagrangian, which leads to a gravitational field equation of order $2 k+4$. Some preliminary results to this type of equations are already due to Buchdahl [38]. For $k=1$, the cosmological consequences of the corresponding sixth-order field equations are discussed in [66] and [75].

In the present chapter we deduce the Newtonian limit following from this higher order field equation. The Newtonian limit of General Relativity Theory is the usual Newtonian theory. From the general structure of the linearized higher-order field equation one can expect that for this higherorder gravity the far field of the point mass in the Newtonian limit is the Newtonian potential plus a sum of different Yukawa terms. And just this form is that one discussed in connection with the fifth force. Here we are interested in the details of this connection between higher-order gravity and the lengths and coefficients in the corresponding Yukawa terms.

Let us start with the Lagrangian

$$
\mathcal{L}=\left(R+\sum_{k=0}^{p} a_{k} R \square^{k} R\right) \cdot \sqrt{-g}, \quad a_{p} \neq 0
$$


In our considerations we will assume that for the gravitational constant $G$ and for the speed of light $c$ it holds $G=c=1$. This only means a special choice of units. In eq. (3.56), $R$ denotes the curvature scalar, $\square$ the D'Alembertian, and $g$ the determinant of the metric. Consequently, the coefficient $a_{k}$ has the dimension "length to the power $2 k+2$ ".

The starting point for the deduction of the field equation is the principle of minimal action. A necessary condition for it is the stationarity of the action:

$$
-\frac{\delta \mathcal{L}}{\delta g_{i j}}=8 \pi T^{i j} \sqrt{-g}
$$

where $T^{i j}$ denotes the energy-momentum tensor. The explicit equations for

$$
P^{i j} \sqrt{-g}=-\frac{\delta \mathcal{L}}{\delta g_{i j}}
$$

are given in [82]. Here we only need the linearized field equation. It reads, cf. $[75]$

$$
P^{i j} \equiv R^{i j}-\frac{R}{2} g^{i j}+2 \sum_{k=0}^{p} a_{k}\left[g^{i j} \square^{k+1} R-\square^{k} R^{; i j}\right]=8 \pi T^{i j},
$$

and for the trace it holds:

$$
g_{i j} \cdot P^{i j}=-\frac{n-1}{2} R+2 n \sum_{k=0}^{p} a_{k}\left[g^{i j} \square^{k+1} R\right]=8 \pi T .
$$

$n$ is the number of spatial dimensions; the most important application is of cause $n=3$. From now on we put $n=3$.

\subsection{The Newtonian limit in higher-order gravity}

The Newtonian limit is the weak-field static approximation. So we use the linearized field equation and insert a static metric and an energy-momentum tensor

$$
T_{i j}=\delta_{i}^{0} \quad \delta_{j}^{0} \rho, \quad \rho \geq 0
$$

into eq. (3.59). 
Without proof we mention that the metric can be brought into spatially conformally flat form, and so we may use

$$
\begin{array}{r}
g_{i j}=\eta_{i j}+f_{i j}, \\
\eta_{i j}=\operatorname{diag}(1,-1,-1,-1) \quad \text { and } \\
f_{i j}=\operatorname{diag}(-2 \Phi,-2 \Psi,-2 \Psi,-2 \Psi) .
\end{array}
$$

Then the metric equals

$$
d s^{2}=(1-2 \Phi) d t^{2}-(1+2 \Psi)\left(d x^{2}+d y^{2}+d z^{2}\right)
$$

where $\Phi$ and $\Psi$ depend on $x, y$ and $z$. Linearization means that the metric $g_{i j}$ has only a small difference to $\eta_{i j}$; quadratic expressions in $f_{i j}$ and its partial derivatives are neglected. We especially consider the case of a point mass. In this case it holds: $\Phi=\Phi(r), \Psi=\Psi(r)$, with

$$
r=\sqrt{x^{2}+y^{2}+z^{2}}
$$

because of spherical symmetry and $\rho=m \delta$. Using these properties, we deduce the field equation and discuss the existence of solutions of the above mentioned type.

At first we make some helpful general considerations: The functions $\Phi$ and $\Psi$ are determined by eq. (3.59) for $i=j=0$ and the trace of eq. (3.59). If these two equations hold, then all other component-equations are automatically satisfied. For the 00-equation we need $R_{00}$ :

$$
R_{00}=-\Delta \Phi
$$

Here, the Laplacian is given is as usual by

$$
\Delta=\frac{\partial^{2}}{\partial x^{2}}+\frac{\partial^{2}}{\partial y^{2}}+\frac{\partial^{2}}{\partial z^{2}}
$$

For the inverse metric we get

$$
g^{i j}=\operatorname{diag}(1 /(1-2 \Phi),-1 /(1+2 \Psi),-1 /(1+2 \Psi),-1 /(1+2 \Psi))
$$


and $1 /(1-2 \Phi)=1+2 \Phi+h(\Phi)$, where $h(\Phi)$ is quadratic in $\Phi$ and vanishes after linearization. So we get

$$
g^{i j}=\eta^{i j}-f^{i j}
$$

In our coordinate system, $f^{i j}$ equals $f_{i j}$ for all $i, j$. For the curvature scalar we get

$$
R=2(2 \Delta \Psi-\Delta \Phi) .
$$

Moreover, we need expressions of the type $\square^{k} R$. $\square R$ is defined by $\square R=$ $R_{; i j} g^{i j}$, where ";" denotes the covariant derivative. Remarks: Because of linearization we may replace the covariant derivative with the partial one. So we get

$$
\square^{k} R=(1)^{k} 2\left(-\Delta^{k+1} \Phi+2 \Delta^{k+1} \Psi\right)
$$

and after some calculus

$$
-8 \pi \rho=\Delta \Phi+\Delta \Psi .
$$

We use eq. (3.71) to eliminate $\Psi$ from the system. So we get an equation relating $\Phi$ and $\rho=m \delta$.

$$
-4 \pi\left(\rho+8 \sum_{k=0}^{p} a_{k}(-1)^{k} \Delta^{k+1} \rho\right)=\Delta \Phi+6 \sum_{k=0}^{p} a_{k}(-1)^{k} \Delta^{k+2} \Phi .
$$

In spherical coordinates it holds

$$
\Delta \Phi=\frac{2}{r} \Phi_{, r}+\Phi_{, r r},
$$

because $\Phi$ depends on the radial coordinate $r$ only.

We apply the following lemma: In the sense of distributions it holds

$$
\Delta\left(\frac{1}{r} e^{-r / l}\right)=\frac{1}{r l^{2}} e^{-r / l}-4 \pi \delta .
$$

Now we are ready to solve the whole problem. We assume

$$
\Phi=\frac{m}{r}\left(1+\sum_{i=0}^{q} c_{i} \exp \left(-r / l_{i}\right)\right), \quad l_{i}>0 .
$$


Without loss of generality we may assume $l_{i} \neq l_{j}$ for $i \neq j$. Then eq. (3.72) together with that lemma yield

$$
\begin{aligned}
8 \pi \sum_{k=0}^{p} a_{k}(-1)^{k} \Delta^{k+1} \delta= & \sum_{i=0}^{q}\left(\frac{c_{i}}{t_{i}}+6 \sum_{k=0}^{p} a_{k}(-1)^{k} \frac{c_{i}}{t_{i}^{k+2}}\right) \frac{1}{r l^{2}} e^{-r / l_{i}} \\
& -4 \pi \sum_{i=0}^{q}\left(c_{i}+6 \sum_{k=0}^{p} a_{k}(-1)^{k} \frac{c_{i}}{t_{i}^{k+1}}\right) \delta \\
& +24 \pi \sum_{k=0}^{p} \sum_{j=k}^{p} \sum_{i=0}^{p} c_{i} a_{j}(-1)^{j+1} \frac{1}{t_{i}^{j-k}} \Delta^{k+1} \delta
\end{aligned}
$$

where $t_{i}=l_{i}^{2} ;$ therefore also $t_{i} \neq t_{j}$ for $i \neq j$. This equation is equivalent to the system

$$
\begin{aligned}
\sum_{i=0}^{q} c_{i} & =1 / 3, \\
\sum_{i=0}^{q} \frac{c_{i}}{t_{i}^{s}}=0, & s=1, \ldots p \\
t_{i}^{p+1}+6 \sum_{k=0}^{p} a_{k}(-1)^{k} t_{i}^{p-k} & =0, \quad i=0, \ldots q .
\end{aligned}
$$

From eq. (3.79) we see that the values $t_{i}$ represent $q+1$ different solutions of one polynomial. This polynominal has the degree $p+1$. Therefore $q \leq p$.

Now we use eqs. (3.77) and (3.78) . They can be written in matrix form as

$$
\left(\begin{array}{c}
1 \ldots 1 \\
1 / t_{0} \ldots 1 / t_{q} \\
\ldots \\
1 / t_{0}^{p} \ldots 1 / t_{q}^{p}
\end{array}\right) \cdot\left(\begin{array}{c}
c_{0} \\
c_{1} \\
\ldots \\
c_{q}
\end{array}\right)=\left(\begin{array}{c}
1 / 3 \\
0 \\
\ldots \\
0
\end{array}\right)
$$

Here, the first $q+1$ rows form a regular matrix, the Vandermonde matrix. Therefore, we get

$$
1 / t_{i}^{j}=\sum_{k=0}^{q} \lambda_{j k} / t_{i}^{k} \quad j=q+1, \ldots p
$$

with certain coefficients $\lambda_{j k}$ i.e., the remaining rows depend on the first $q+1$ ones. If $\lambda_{j 0} \neq 0$ then the system has no solution. So $\lambda_{j 0}=0$ for all $q+1 \leq$ 
$j \leq p$. But for $q<p$ we would get

$$
1 / t_{i}^{q}=\sum_{k=1}^{q} \lambda_{q+1 k} / t_{i}^{k-1}
$$

and this is a contradiction to the above stated regularity. Therefore $p$ equals $q$. The polynomial in (14) may be written as

$$
6 \cdot\left(\begin{array}{c}
11 / t_{0} \ldots 1 / t_{0}^{p} \\
\ldots \\
11 / t_{p} \ldots 1 / t_{p}^{p}
\end{array}\right) \cdot\left(\begin{array}{c}
a_{0} \\
\ldots \\
(-1)^{p} a_{p}
\end{array}\right)=\left(\begin{array}{c}
-t_{0} \\
\ldots \\
-\tau_{p}
\end{array}\right)
$$

This matrix is again a Vandermonde one, i.e., there exists always a unique solution $\left(a_{0}, \ldots a_{p}\right)$, which are the coefficients of the quantum corrections to the Einstein equation, such that the Newtonian limit of the corresponding gravitational field equation is a sum of Newtonian and Yukawa potential with

prescribed lengths $l_{i}$. A more explicit form of the solution is given in section 3.9 .

\subsection{Discussion of the weak-field limit}

Let us give some special examples of the deduced formulas of the Newtonian limit of the theory described by the Lagrangian (3.56). If all the $a_{i}$ vanish we get of course the usual Newton theory

$$
\Phi=\frac{m}{r}, \quad \Delta \Phi=-4 \pi \delta .
$$

$\Phi$ and $\Psi$ refer to the metric according to eq. (3.63). For $p=0$ we get for $a_{0}<0$

$$
\Phi=\frac{m}{r}\left[1+\frac{1}{3} e^{-r / \sqrt{-6 a_{0}}}\right]
$$

cf. [59] and

$$
\Psi=\frac{m}{r}\left[1-\frac{1}{3} e^{-r / \sqrt{-6 a_{0}}}\right] .
$$

For $a_{0}>0$ no Newtonian limit exists.

For $p=1$, i.e., the theory following from sixth-order gravity

$$
\mathcal{L}=\left(R+a_{0} R^{2}+a_{1} R \square R\right) \sqrt{-g},
$$


we get, see [81]

$$
\Phi=\frac{m}{r}\left[1+c_{0} e^{-r / l_{0}}+c_{1} e^{-r / l_{1}}\right]
$$

and

$$
\Psi=\frac{m}{r}\left[1-c_{0} e^{-r / l_{0}}-c_{1} e^{-r / l_{1}}\right]
$$

where

$$
c_{0,1}=\frac{1}{6} \mp \frac{a_{0}}{2 \sqrt{9 a_{0}^{2}+6 a_{1}}}
$$

and

$$
l_{0,1}=\sqrt{-3 a_{0} \pm \sqrt{9 a_{0}^{2}+6 a_{1}}}
$$

This result is similar in structure but has different coefficients as in fourthorder gravity with included square of the Weyl tensor in the Lagrangian.

The Newtonian limit for the degenerated case $l_{0}=l_{1}$ can be obtained by a limiting procedure as follows: As we already know $a_{0}<0$, we can choose the length unit such that $a_{0}=-1 / 3$. The limiting case $9 a_{0}^{2}+6 a_{1} \rightarrow 0$ may be expressed by $a_{1}=-1 / 6+\varepsilon^{2}$. After linearization in $\varepsilon$ we get:

$$
l_{i}=1 \pm \sqrt{3 / 2} \varepsilon c_{i}=1 / 6 \pm 1 /(6 \sqrt{6} \varepsilon)
$$

and applying the limit $\varepsilon \rightarrow 0$ to the corresponding fields $\Phi$ and $\Psi$ we get

$$
\begin{gathered}
\Phi=m / r\left\{1+(1 / 3+r / 6) e^{-r}\right\} \\
\Psi=m / r\left\{1-(1 / 3+r / 6) e^{-r}\right\} .
\end{gathered}
$$

For the general case $p>1$, the potential is a complicated expression, but some properties are explicitly known, these hold also for $p=0,1$. One gets

$$
\Phi=m / r\left(1+\sum_{i=0}^{p} c_{i} \exp \left(-r / l_{i}\right)\right)
$$

and

$$
\Psi=m / r\left(1-\sum_{i=0}^{p} c_{i} \exp \left(-r / l_{i}\right)\right)
$$

where $\sum c_{i}=1 / 3 ; \sum$ means $\sum_{i=0}^{p}$ and $l_{i}$ and $c_{i}$ are, up to permutation of indices, uniquely determined by the Lagrangian. 
There exist some inequalities between the coefficients $a_{i}$, which must be fulfilled in order to get a physically acceptable Newtonian limit. By this phrase we mean that besides the above conditions, additionally the fields $\Phi$ and $\Psi$ vanish for $r \rightarrow \infty$ and that the derivatives $d \Phi / d r$ and $d \Psi / d r$ behave like $O\left(1 / r^{2}\right)$. These inequalities express essentially the fact that the $l_{i}$ are real, positive, and different from each other. The last of these three conditions can be weakened by allowing the $c_{i}$ to be polynomials in $r$ instead of being constants, cf. the example with $p=1$ calculated above.

The equality $\sum c_{i}=1 / 3$ means that the gravitational potential is unbounded and behaves, up to a factor $4 / 3$, like the Newtonian potential for $r \approx 0$. The equation $\Phi+\Psi=2 m / r$ enables us to rewrite the metric as

$$
d s^{2}=(1-2 \theta)\left[(1-2 m / r) d t^{2}-(1+2 m / r)\left(d x^{2}+d y^{2}+d z^{2}\right)\right],
$$

which is the conformally transformed linearized Schwarzschild metric with the conformal factor $1-2 \theta$, where

$$
\theta=\frac{m}{r} \sum c_{i} e^{-r / l_{i}}
$$

can be expressed as functional of the curvature scalar, this is the linearized version of the conformal transformation theorem. For an arbitrary matter configuration the gravitational potential can be obtained by the usual integration procedure.

\subsection{A homogeneous sphere}

For general $p$ and characteristic lengths $l_{i}$ fulfilling $0<l_{0}<l_{1}<\ldots<l_{p}$ we write the Lagrangian as

$$
\begin{aligned}
\mathcal{L}= & R-\frac{R}{6}\left[\left(l_{0}^{2}+\ldots+l_{p}^{2}\right) R+\left(l_{0}^{2} l_{1}^{2}+l_{0}^{2} l_{2}^{2} \ldots+l_{p-1}^{2} l_{p}^{2}\right) \square R+\right. \\
& \left.\left(l_{0}^{2} l_{1}^{2} l_{2}^{2}+\ldots+l_{p-2}^{2} l_{p-1}^{2} l_{p}^{2}\right) \square^{2} R+\ldots+l_{0}^{2} \cdot l_{1}^{2} \cdot \ldots \cdot l_{p}^{2} \square^{p} R\right]
\end{aligned}
$$

the coefficients in front of $\square^{i} R$ in this formula read

$$
\sum_{0 \leq j_{0}<j_{1}<\ldots<j_{i} \leq p} \prod_{m=0}^{i} l_{j_{m}}^{2} .
$$


Using this form of the Lagrangian, the gravitational potential of a point mass reads

$$
\begin{aligned}
& \Phi=\frac{m}{r}\left[1+\frac{1}{3} \sum_{i=0}^{p}(-1)^{i+1} \prod_{j \neq i}\left|\frac{l_{j}^{2}}{l_{i}^{2}}-1\right|^{-1} e^{-r / l_{i}}\right], \\
& \Psi=\frac{m}{r}\left[1-\frac{1}{3} \sum_{i=0}^{p}(-1)^{i+1} \prod_{j \neq i}\left|\frac{l_{j}^{2}}{l_{i}^{2}}-1\right|^{-1} e^{-r / l_{i}}\right] .
\end{aligned}
$$

For a homogeneous sphere of radius $r_{0}$ and mass $m$ we get

$$
\Phi=\frac{m}{r}\left[1+\frac{1}{r_{0}^{3}} \sum_{i=0}^{p} e^{-r / l_{i}} l_{i}^{2} \tilde{c}_{i}\left(r_{0} \cosh \left(r_{0} / l_{i}\right)-l_{i} \sinh \left(r_{0} / l_{i}\right)\right)\right],
$$

where

$$
\tilde{c}_{i}=(-1)^{i+1} \prod_{j \neq i}\left|\frac{l_{j}^{2}}{l_{i}^{2}}-1\right|^{-1}
$$

\section{Acknowledgement}

I thank the Organizers of the 42nd Karpacz Winter School for the kind invitation to present these lectures, and I thank several colleagues, especially S. Capozziello, M. Francaviglia, A. Mazumdar, S. Odintsov, V. Sahni, A. Starobinsky and L. Urena for useful comments to the first version of this paper.

\section{Bibliography}

[1] K. Lanczos: A remarkable property of the Riemann - Christoffel tensor in four dimensions. Annals of Math. 39 (1938) 842-850.

[2] H. Weyl: Gravitation und Elektrizität. Sitzungsber. Preuss. Akad. d. Wiss. Teil 1 (1918) 465-480.

[3] R. Utiyama, B. de Witt: Renormalization of a classical gravitational field interacting with quantized matter fields, J. Math. Phys. 3 (1962) 608.

[4] K. Stelle: Renormalization of higher-derivative quantum gravity. Phys. Rev. D 16 (1977) 953-969. 
[5] H. Weyl: Reine Infinitesimalgeometrie. Mathemat. Zeitschr. 2 (1918) 384-411.

[6] H. Weyl: Eine neue Erweiterung der Relativitätstheorie. Ann. d. Phys. Leipz. (4) 59 (1919) 101-133.

[7] H. Weyl: Elektrizität und Gravitation. Physik. Zeitschr. 21 (1920) 649-650.

[8] H. Weyl: Physik. Zeitschr. 22 (1921) 473-480.

[9] H. Weyl: Electricity and Gravitation. Nature 106 (1921) 800-802.

[10] H. Weyl: Raum, Zeit, Materie, (Berlin, Springer 1923).

[11] E. Schrödinger: Über eine bemerkenswerte Eigenschaft der Quantenbahnen eines einzelnen Elektrons. Zeitschr. f. Physik 12 (1923) 13-23.

[12] H. Weyl: Elektron und Gravitation I. Zeitschr. f. Physik 56 (1929) 330-352.

[13] F. London: Zeitschr. f. Physik 42 (1927) 375-389.

[14] V. Vizgin: Einstein, Hilbert, Weyl: Genesis des Programms der einheitlichen geometrischen Feldtheorien. NTM-Schriftenr. Leipzig 21 (1984) 23-33.

[15] A. Einstein: Addendum to [2]; p. 478.

[16] P. Dirac: Long range forces and broken symmetries. Proc. R. Soc. Lond. A 333 (1973) 403-418.

[17] E. Reichenbächer: Die Eichinvarianz des Wirkungsintegrals und die Gestalt der Feldgleichungen in der Weylschen Theorie. Z. f. Physik 22 (1924) 157-169.

[18] R. Weitzenböck: Über die Wirkungsfunktion in der Weylschen Physik 1, 2, 3. Sitzungsber. Akad. d. Wiss. Wien, Math.-naturwiss. Kl. Abt. IIa, 129 (1920) 683-696; 697-703; 130 (1921) 15-23.

[19] W. Pauli: Zur Theorie der Gravitation und der Elektrizität von Hermann Weyl. Physik. Zeitschr. 20 (1919) 457-467.

[20] W. Pauli: Merkurperihelbewegung und Strahlenablenkung in Weyls Gravitationstheorie. Berichte d. Deutschen Phys. Ges. 21 (1919) 742-750.

[21] F. Jüttner: Beiträge zur Theorie der Materie. Math. Annalen 87 (1922) 270-306.

[22] W. Pauli: Relativitätstheorie, Enc. math. Wiss. Bd. 5, Teil 2, S. 543-775 (Leipzig, Teubner Verl. 1922). 
[23] R. Bach: Zur Weylschen Relativitätstheorie und der Weylschen Erweiterung des Krümmungsbegriffs. Math. Zeitschr. 9 (1921) 110-135.

[24] A. Einstein: Eine naheliegende Ergänzung des Fundamentes der allgemeinen Relativitätstheorie. Sitzungsbericht. Preuss. Akad. d. Wiss. Teil 1, (1921) 261-264.

[25] E. Reichenbächer: Eine neue Erklärung des Elektromagnetismus, Z. f. Physik 13 (1923) 221-240.

[26] U. Kakinuma: On the structure of an electron, Part I, II. Proc. Phys.Math. Soc. Japan Ser. 3, I (1928) 235-242; II (1929) 1-11.

[27] C. Lanczos: Elektromagnetismus als natürliche Eigenschaft der Riemannschen Geometrie. Zeitschr. f. Physik 73 (1932) 147-168.

[28] C. Lanczos: Zum Auftreten des Vektorpotentials in der Riemannschen Geometrie. Zeitschr. f. Physik 75 (1932) 63-77.

[29] C. Lanczos: Electricity as a natural property of Riemannian geometry. Phys. Rev. 39 (1932) 716-736.

[30] C. Lanczos: Ein neuer Aufbau der Weltgeometrie. Zeitschr. f. Physik 96 (1935) 76-106.

[31] C. Lanczos: Matter waves and Electricity. Phys. Rev. 61 (1942) 713720.

[32] C. Lanczos: Lagrangian multipliers and Riemannian spaces. Rev. Mod. Phys. 21 (1949) 497-502.

[33] C. Lanzcos: Electricity and General Relativity. Rev. Mod. Phys. 29 (1957) 337-350.

[34] C. Lanczos: Quadratic action principle of Relativity. J. Math. Phys. 10 (1969) 1057-1065.

[35] V. Vizgin: Hermann Weyl, die Göttinger Tradition der mathematischen Physik und einheitliche Feldtheorien. Wiss. Zeitschr. d. E.-M.-Arndt-Univ. Greifswald, Math.-naturwiss. Reihe 33 (1984) 57-60.

[36] P. Bergmann: Introduction to the theory of relativity (New York, Prentice Hall 1942).

[37] H. Buchdahl: On functionally constant invariants of the Riemann tensor. Proc. Cambr. Philos. Soc. 68 (1970) 179-185.

[38] H. Buchdahl: Über die Variationsableitung von Fundamentalinvarianten beliebig hoher Ordnung. Acta Mathematica 85 (1951) 63-72. 
[39] H. Buchdahl: On the Hamilton derivatives arising from a class of gaugeinvariant action principles in a $W_{n}$. J. Lond. Math. Soc. 26 (1951) 139-149. [40] H. Buchdahl: An identity between the Hamiltonian derivatives of certain fundamental invariants in a $W_{4}$. J. Lond. Math. Soc. 26 (1951) 150-152.

[41] H. Buchdahl: On the gravitational field equations arising from the square of the Gaussian curvature. Nuovo Cim. 23 (1962) 141-156.

[42] H. Buchdahl: The Hamiltonian derivatives of a class of fundamental invariants. Quart. J. Math. Oxford 19 (1948) 150.

[43] H. Buchdahl: A special class of solutions of the equations of the gravitational field arising from certain gauge-invariant action principles. Proc. Nat. Acad. Sci. USA 34 (1948) 66-68.

[44] H. Buchdahl: Reciprocal static metrics and non-linear Lagrangians. Tensor 21 (1970) 340-344.

[45] H. Buchdahl: Quadratic Lagrangians and static gravitational fields. Proc. Cambr. Philos. Soc. 74 (1973) 145-148.

[46] H. Buchdahl: Non-linear lagrangians and cosmological theory. Monthly Not. R. Astron. Soc. 150 (1970) 1-8.

[47] H. Buchdahl: The field equations generated by the square of the scalar curvature: solutions of the Kasner type. J. Phys. A 11 (1978) 871-876.

[48] H. Buchdahl: On a set of conform-invariant equations of the gravitational field. Proc. Edinburgh Math. Soc. 10 (1953) 16-20.

[49] H. Buchdahl: Remark on the equation $\delta R^{2} / \delta g^{i j}=0$. Intern. J. Theor. Phys. 17 (1978) 149-151.

[50] A. Eddington: Relativity Theory (in German) (Berlin, Springer 1925).

[51] E. Schrödinger: Space-time structure (Cambridge University Press 1950).

[52] C. Gregory: Non-linear invariants and the problem of motion. Phys. Rev. 72 (1947) 72-75.

[53] E. Pechlaner, R. Sexl: On quadratic Lagrangians in General Relativity. Commun. Math. Phys. 2 (1966) 165-173.

[54] A. D. Sakharov: Vakuumnye kvantovye fluktuacii v iskrivlennom prostranstve i teoria gravitacii. Dokl. Akad. Nauk SSSR 177 (1967) 70-71; reprinted with comments in Gen. Relat. Grav. 32 (2000) 365.

[55] H.-J. Treder: Ann. Phys. Leipz. 32 (1975) 383-400. 
[56] B. Ivanov: Cosmological solution with string correction. Phys. Lett. B 198 (1987) 438.

[57] D. Hochberg, T. Shimada: Ambiguity in determining the effective action for string-corrected Einstein gravity. Progr. Theor. Phys. 78 (1987) 680.

[58] V. Müller, H.-J. Schmidt: On Bianchi type I vacuum solutions in $R+R^{2}$ theories of gravitation. I. The isotropic case. Gen. Relat. Grav. 17 (1985) 769-781.

[59] Stelle, K.: Classical gravity with higher derivatives. Gen. Relat. Grav. 9 (1978) 353-371.

[60] N. Mio: Experimental test of the law of gravitation at small distances. Phys. Rev. D 36 (1987) 2321.

[61] F. Stacey, G. Tuck, G. Moore: Quantum Gravity: Observational constraints on a pair of Yukawa terms. Phys. Rev. D 36 (1987) 2374.

[62] M. Ander, M. Nieto: Possible resolution of the Brookhaven and Eötvös experiments. Phys. Rev. Lett. 60 (1988) 1225.

[63] L. Amendola, A. Battaglia Mayer, S. Capozziello, S. Gottlöber, V. Müller, F. Occhionero, H.-J. Schmidt: Class. Quant. Grav. 10 (1993) L43.

[64] J. Barrow: Phys. Lett. B 180 (1986) 335; Phys. Lett. B 187 (1987) 12. [65] A. Battaglia Mayer, H.-J. Schmidt: Class. Quant. Grav. 10 (1993) 2441.

[66] A. Berkin, K. Maeda: Phys. Lett. B 245 (1990) 348; Phys. Rev. D 44 (1991) 1691.

[67] J. Bičák: Lect. Notes Phys. 540 (2000) 1-126; gr-qc/0004016.

[68] G. Bicknell: J. Phys. A 7 (1974) 341. 1061.

[69] S. Capozziello, G. Lambiase: Gen. Relat. Grav. 32 (2000) 295, 673.

[70] A. Coley, R. Tavakol: Gen. Relat. Grav. 24 (1992) 835.

[71] S. Cotsakis, J. Demaret, Y. De Rop, L. Querella: Phys. Rev. D 48 (1993) 4595.

[72] D. Coule: Phys. Rev. D 62 (2000) 124010, gr-qc/0007037; Class. Quant. Grav. 12 (1995) 455, gr-qc/9408026.

[73] G. Ellis: Ann. Rev. Astron. Astrophys. 22 (1984) 157; J. Math. Phys. 8 (1967) 1171. 
[74] S. Gottlöber, V. Müller, H.-J. Schmidt, A. A. Starobinsky: Int. J. Mod. Phys. D 1 (1992) 257.

[75] S. Gottlöber, H.-J. Schmidt, A. A. Starobinsky: Class. Quantum Grav. 7 (1990) 893.

[76] A. Jakubiec, J. Kijowski: Phys. Rev. D 37 (1988) 1406; J. Math. Phys. 30 (1989) 1073.

[77] K. Maeda: Phys. Rev. D 37 (1988) 858; Phys. Rev. D 39 (1989) 3159. [78] V. Müller, H.-J. Schmidt: Gen. Relat. Grav. 17 (1985) 769; Gen. Relat. Grav. 21 (1989) 489.

[79] V. Müller, H.-J. Schmidt, A. Starobinsky: Phys. Lett. B 202 (1988) 198.

[80] I. Prigogine, J. Geheniau, E. Gunzig, P. Nardone: Proc. Nat. Acad. Sci. 85(1988) 7428; Gen. Relat. Grav. 21 (1989) 767; Int. J. Theor. Phys. 28 (1989) 927.

[81] I. Quandt, H.-J. Schmidt: Astron. Nachr. 312 (1991) 97; gr-qc/0109005. [82] H.-J. Schmidt: Astron. Nachr. 303 (1982) 227, gr-qc/0105104; Astron. Nachr. 303 (1982) 283, gr-qc/0105105; Ann. Phys. (Leipz.) 41 (1984) 435, gr-qc/0105108; Astron. Nachr. 306 (1985) 67, gr-qc/0105107; Astron. Nachr. 307 (1986) 339, gr-qc/0106037; Astron. Nachr. 308 (1987) 183, gr-qc/0106035; Class. Quant. Grav. 5 (1988) 233; Phys. Lett. B 214 (1988) 519; Class. Quant. Grav. 6 (1989) 557; Phys. Rev. D 50 (1994) 5452, gr-qc/0109006; Phys. Rev. D 52 (1995) 6198, gr-qc/0106034; Class. Quantum Grav. 7 (1990) 1023; Phys. Rev. D 49 (1994) 6354; Phys. Rev. D 54 (1996) 7906, gr-qc/9404038; Grav. Cosmol. 3 (1997) 185, gr-qc/9709071; Int. J. Theor. Phys. 37 (1998) 691, gr-qc/9512007.

[83] H.-J. Schmidt, V. Müller: Gen. Relat. Grav. 17 (1985) 971.

[84] A. A. Starobinsky, H.-J. Schmidt: Class. Quant. Grav. 4 (1987) 695.

[85] B. Whitt: Phys. Lett. B 145 (1984) 176.

[86] M. Amarzguioui, O. Elgaroy, D.F. Mota, T. Multamaki: Cosmological constraints on $f(R)$ gravity theories within the Palatini approach, astroph/0510519.

[87] S. Capozziello, V. F. Cardone, A. Troisi: Reconciling dark energy models with $f(R)$ theories, astro-ph/0501426, Phys. Rev. D 71 (2005) 043503.

[88] S. Carloni, P. Dunsby, S. Capozziello, A. Troisi: Cosmological dynamics 
of $R^{n}$ gravity, gr-qc/0410046, Class. Quant. Grav. 22 (2005) 4839.

[89] T. Clifton, J. Barrow: The existence of Gödel, Einstein and de Sitter Universes, Phys. Rev. D 72 (2005) 123003, gr-qc/0511076.

[90] T. Clifton, J. Barrow: The power of General Relativity, Phys. Rev. D 72 (2005) 103005.

[91] D. Coule: Quantum Cosmological Models, gr-qc/0412026, Class. Quant. Grav. 22 (2005) R125-R166.

[92] A. Rendall: Intermediate inflation and the slow-roll approximation, Class. Quant. Grav. 22 (2005) 1655.

[93] A. Sanyal, B. Modak, C. Rubano, E. Piedipalumbo: Noether symmetry in the higher order gravity theory, Gen. Relat. Grav. 37 (2005) 407.

[94] A. Sanyal: Hamiltonian formulation of curvature squared action, Gen. Relat. Grav. 37 (2005) 1957.

[95] F. Schunck, F. Kusmartsev and E. Mielke: Dark matter problem and effective curvature Lagrangians, Gen. Relat. Grav. 37 (2005) 1427.

[96] G. Allemandi, M. Capone, S. Capozziello: Conformal aspects of Palatini approach in Extended Theories of Gravity, Gen. Relat. Grav. 38 (2006) 33.

[97] J. Barrow, T. Clifton: Exact cosmological solutions of scale-invariant gravity theories, Class. Quantum Grav. 23 (2006) L1, gr-qc/0509085.

[98] J. Barrow, S. Hervik: Anisotropically Inflating Universes, Phys. Rev. D 73 (2006) 023007, gr-qc/0511127.

[99] K. A. Bronnikov, M. S. Chernakova: Generalized theories of gravity and conformal continuations, Grav. Cosmol. 11 (2005) 305; gr-qc/0601123.

[100] V. Sahni, L. A. Kofman: Some self-consistent solutions of the Einstein equations with one-loop quantum gravitational corrections, Phys. Lett. A 117 (1986) 275-278.

[101] I. Moss and V. Sahni: Anisotropy in the chaotic inflationary universe, Phys. Lett. B 178 (1986) 159-162

[102] Octavio Obregon, L. Arturo Urena-Lopez, Franz E. Schunck: Oscillatons formed by non linear gravity, Phys. Rev. D 72 (2005) 024004; grqc/0404012.

[103] A. A. Starobinsky: A new type of isotropic cosmological model without singularity, Phys. Lett. B 91 (1980) 99.

[104] A. A. Starobinsky: Isotropization of arbitrary cosmological expansion 
given by an effective cosmological constant, Sov. Phys. JETP Lett. 37 (1983) 66.

[105] T. Biswas, A. Mazumdar, W. Siegel: Bouncing Universes in Stringinspired Gravity, hep-th/0508194 (2005).

[106] S. Nojiri, S. D. Odintsov: Introduction to Modified Gravity and Gravitational Alternative for Dark Energy, hep-th/0601213, v.3 (2006).

[107] G. Cognola, E. Elizalde, S. Nojiri, S. D. Odintsov, S. Zerbini: Dark energy in modified Gauss-Bonnet gravity: late-time acceleration and the hierarchy problem, hep-th/0601008 (2006).

[108] G. Allemandi, M. Capone, S. Capozziello: Conformal aspects of Palatini approach in Extended Theories of Gravity, Gen. Rel. Grav. 38 (2006) 33. [109] S. Capozziello, V. F. Cardone, E. Piedipalumbo, C. Rubano: Dark energy exponential potential models as curvature quintessence, Class. Quant. Grav. 23 (2006) 1205-1216; astro-ph/0507438. 